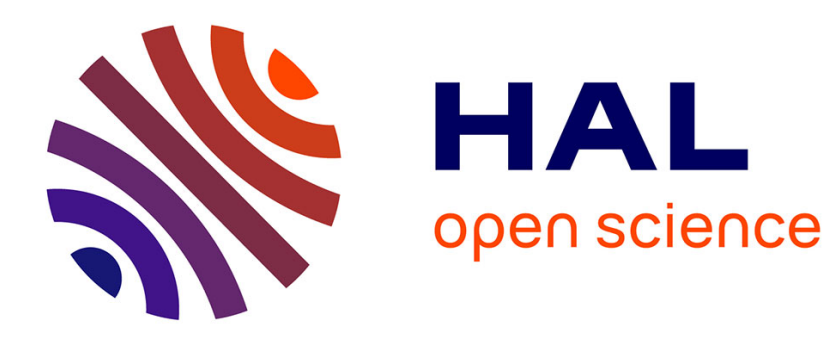

\title{
Informational Branching Universe
}

Pierre Uzan

\section{To cite this version:}

Pierre Uzan. Informational Branching Universe. Foundations of Science, 2010. hal-03458836

\section{HAL Id: hal-03458836 \\ https://hal.science/hal-03458836}

Submitted on 30 Nov 2021

HAL is a multi-disciplinary open access archive for the deposit and dissemination of scientific research documents, whether they are published or not. The documents may come from teaching and research institutions in France or abroad, or from public or private research centers.
L'archive ouverte pluridisciplinaire HAL, est destinée au dépôt et à la diffusion de documents scientifiques de niveau recherche, publiés ou non, émanant des établissements d'enseignement et de recherche français ou étrangers, des laboratoires publics ou privés. 


\section{Informational Branching Universe}

“...all things physical are

information-theoretic in origin and

this is a participatory universe."

J. A. Wheeler (1990)

This paper suggests an epistemic interpretation of Belnap's branching space-times theory based on Everett's relative state formulation of the measurement operation in quantum mechanics. The informational branching models of the universe are evolving structures defined from a partial ordering relation on the set of memory states of the impersonal observer. The totally ordered set of their information contents defines a linear "time" scale to which the decoherent alternative histories of the informational universe can be referred -which is quite necessary for assigning them a probability distribution.

The "historical" state of a physical system is represented in an appropriate extended Hilbert space and an algebra of multi-branch operators is developed. An age operator computes the informational depth of historical states and its standard deviation can be used to provide a universal information/energy uncertainty relation. An information operator computes the encoding complexity of historical states, the rate of change of its average value accounting for the process of correlation destruction inherent to the branching dynamics. In the informational branching models of the universe, the asymmetry of phenomena in nature appears as a mere consequence of the subject's activity of measuring, which defines the flow of time-information.

Key words: branching space-times, information, algorithmic complexity, historical states. 


\section{Introduction}

Branching space-times theory aims to reconcile indeterminism and relativity by modelling indeterminism as the branching of relativistic space-times (Belnap 2003) (Müller 2001). Branching space-times models are based on the causal relativistic (partial) ordering relation on the set of all possible point-events. One of the main interests of interpreting indeterminism by the branching of relativistic space-time is, of course, to reconcile relativity and quantum indeterminism.

As a matter of fact, branching space-times (BST) models have been used to clarify quantum concepts, like those of non-separability and contextuality, by characterizing precisely EPR-like correlations (Belnap 2002) and by reformulating the GHSZ-Mermin theorem (Belnap and Szabó 1996). They have also been used to suggest an interpretation of quantum probabilities in a relativistic space-time arena and to deal with the question of relativistic covariance of state vector reduction (Mc Call 1998). In the area of quantum gravity, the BST models can help to interpret consistently the question of quantum fluctuations of the spacetime geometry in regions on the scale of the Planck length and the question of its topology change (Sharlow 2004). The application of the BST theory to quantum physics may thus prove very successful.

However, paradoxically, BST models are based on an "objectivist" conception of physical reality, where the notion of experimental activity is totally absent. In BST models the set of all "possible" point-events on which the partial ordering relation is defined is a strongly objective reality which is wholly given from an external, Archimedean point of view, independently of any observer, of any observation and of any action. In particular, the role of the measurement operation, which, as shown by long-standing debates on quantum theory (Wheeler and Zurek 1983) (d'Espagnat 1994, chap. 10), constitutes an essential issue in any attempt to understand this theory, is not explicitly addressed. Yet, and in spite of some 
proposals to restore an ontological interpretation of quantum mechanics (Bohm and Hiley 1993) (Girhardi, Rimini and Weber 1986), it seems that, as emphasized for example by d'Espagnat (1979), taking this role into account seriously weakens this "objectivist" worldview: "the doctrine that the world is made up of objects whose existence is independent of human consciousness turns out to be in conflict with quantum mechanics and with the facts established by experiment" (p. 128).

Moreover, and in accordance with the block universe conception of relativity -which is in conflict with daily experience-, it seems that in BST models time does not "flow" and that becoming does not exist unless you postulate, still from the same external point of view, an appropriate process of "branch attrition" (Mc Call 1998). "Point-events" of BST models and the "histories" they constitute have always been there and will always be there, hanging on a World of Platonic Ideas.

As a consequence, in these models the branching of histories is only regarded as a topological property of the objective set of point-events. It is not caused by some interaction between the observer and the physical world but it is postulated as a strongly objective feature of reality that exists for all eternity. The branching of histories relies on a metaphysical concept of indeterminism that reduces the concept of probability only to its propensity-like aspect. Probabilities are then defined in the "austere" language of BST as weightings of “objective" transitions between sets of point events (Belnap 2002) (Placek 2003) (Müller 2005) or they are interpreted as relative proportions of equally "objective" co-existing branches of the universe (Mc Call 1998).

However, it seems that quantum indeterminism first refers to the (inter-)subjective uncertainty about the possible outcomes of measurements performed in a given experimental context, regardless of any possible ontological interpretation of quantum mechanics that could be proposed. According to the uncontroversial minimal interpretation of the formalism of 
quantum mechanics, the wave function (or, more generally, the density operator) that represents the "state" of a quantum system is a contextual predictive tool from which the probabilities of the possible outcomes of measurements performed on this system can be calculated by using Born's rule (Bitbol 1996). Quantum indeterminism cannot thus be identified a priori with a feature of the "objective", independent world -even if such an identification may be suggested a posteriori. This point is corroborated by DestouchesFévrier's analysis, according to which quantum indeterminism is a typical property which concerns predictions of any quantum theory (even determinist, hidden variable theories) whose domain of adequacy contains that of the standard quantum mechanics (DestouchesFevrier 1945).

In this paper the notion of quantum indeterminism will thus be understood from an epistemic point of view which refers to the irreducible, inter-subjective uncertainty relative to measurement operations performed by the impersonal "observer", which can be regarded as an information gathering and utilizing system (IGUS). Accordingly, the branching of the universe will be regarded as a consequence of the splitting of the universal wave function into its different components due to measurement acts, in accordance with Everett's "relative state" formulation of quantum mechanics (Everett 1957), and not as a topological feature of the independent reality. However, according to the present epistemic approach, and contrary to the objectivist "many worlds" interpretation of Everett's original proposal by de Witt (de Witt 1970) where it is assumed that all the outcomes of a measurement (co-)exist in some "world" or in some "branch" of the objective physical universe, the branches of the informational universe do not (co-) "exist" in a strong ontological sense: they only feature the possible evolutions of the IGUS's memory state, only one of them being actualised -a view which is inspired by the Piercean or "antactualist" concept of indeterminism (Prior 1967, chap. VI) (Burgess 1978). In other word, the branching models of the universal information 
process should not be regarded as branching models of the physical universe (or of its spacetime frame) but as representations, in the language of information, of the possible sequences of the IGUS's memory states that correspond to the observation of outcomes of measurements.

As a consequence, the present antactualist concept of indeterminism is not based on the proposals to interpret quantum indeterminism and to derive Born's rule from a quantum decision theory within the many worlds interpretation of Everett's relative state formulation (Wallace 2003) (Saunders 1998) ${ }^{1}$. Indeed, the present epistemic interpretation of quantum indeterminism can find a strong support in Zurek's derivation of Born's rule within the framework of standard quantum mechanics (in a single world), which is based on consideration of the role of the environment and appeals explicitly to the guess of the observer regarding the possible outcomes of a measurement (Zurek 2003, p. 34-39).

To implement this epistemic interpretation of BST theory, I suggest to consider evolving branching models of the informational universe. These models are not the result of an abstract pasting of pre-existing block-universes (Müller 2001) but they are progressively constructed: they feature the universal information process underlying the realization of measurement operations. In this framework the "objective" transitions between point-events of the BST models will be understood as informational transitions between the IGUS's memory states which are triggered by measurement acts -and, generally, by the subject's activity of seeking and finding. This paper will thus focus on this information process, as is the case in Everett's "relative state" formulation (Everett 1957), considering the question of the space-time framework of secondary importance.

\footnotetext{
1 Note, on the other hand, that these very appealing proposals have been recently questioned (Hemmo and Pitowski 2006).
} 
The relevance of such an informational approach can find a confirmation in the advent of quantum information theory. The development of quantum information theory, which governs manipulation and control of quantum systems (Nielsen 1998), and the proposals to reconstruct quantum theory from informational theoretic constraints (Clifton, Bub and Halvorson 2003) (Grinbaum 2004) have led to the idea that information should play a central role in physics. Even more radically, Wheeler (1990) has suggested that the information process constituted by the game of questions posed by the observers (or the measuring devices) and the answers they obtain, the "elementary acts of observer participancy" in his words, is primary and that any item in the physical world derives its ultimate significance from it : “...what we call reality arises in the last analysis from the posing of yes-no questions and the registering of equipment-evoked responses" (p. 5), a view which is illustrated by Wheeler's quotation put as an epigraph to this paper.

Moreover, the informational point of view is not at all incompatible with a logical analysis of the knowledge process on which the notion of "information" relies. If, as explained by Kant (1787) in his Transcendental Aesthetic, "space" and "time" are needed a priori for the perception of any phenomenon and then for any activity of knowledge (p. 53-63), what is exactly required for the perception of distinct objects is "space in general", that is, a spatial representation of them which must not be confused with particular geometric characterisations of space; and, in the same way, perception of successive events presupposes a temporal representation of them which does not at all impose a particular characterisation of time. Consequently, the idea to regard the universal information process as more fundamental than any particular spatio-temporal analysis of the subject's activity is quite defensible. Accordingly, one can say that any particular space-time representation (Newtonian, Minkowskian, Riemannian..... ) can fit with the informational branching models of the universe (IBU models, in short). 
As will be explained in section I, the notion of "information" is used in this paper in its two complementary aspects: 1) as a tool to evaluate what is recorded in the IGUS's memory when he performs a measurement, which refers to a concept of information-knowledge defined in terms of Shannon's statistical information, and, on the other hand, 2) as a tool to evaluate the intrinsic degree of organization of physical objects or that of their "states", which refers to a structural concept of "information" defined in terms of algorithmic complexity. Consequently, the concept of structural information, which is used in this paper to define an information operator and to deal with the question of the arrow of time, must not be confused with the statistical concept of information-knowledge (even if they can be related under certain conditions -see section V.4). Indeed, both concepts of "information" must be used to quantify the information process underlying the action of seeking and finding of the subject of knowledge and action (and none of them have an ontological or a physical status): they are means to deal quantitatively with the interaction subject/world. The evolving models of the informational branching universe will then be defined from a partial ordering relation on the set of the IGUS's memory states of (section II). In order to give a precise meaning to the notion of exhaustive set of decohering histories, a universal, linear "time" frame to which these alternative histories can be referred will be defined from the models of branching spacetime, in terms of information (section III).

Then we shall deal with the question of the "historical" representation of a quantum state and of its temporal evolution in the informational branching models relative to particular physical systems. This question requires the construction of a generalized Hilbert space where an algebra of multi-branch operators can be defined (section IV). The Schrödinger-like evolution is reformulated in the generalized Hilbert spaces (section V.1). In order to deal with the information process relative to the temporal evolution of physical systems, one defines (section V.2) two multi-branch operators: 1) an "age" operator which computes the degree of 
development of their associated informational branching structure (their "size"), and whose standard deviation can be used to define an informational characteristic "duration" of evolution which satisfies a universal information/energy uncertainty relation -this relation reduces, in the ideal case the system can be considered as genuinely isolated, to Heisenberg's time/energy uncertainty relation (section V.3); 2) an information operator, which computes the algorithmic information content of the historical states. Due to the process of destruction of correlation inherent to the branching dynamics, which is caused by the realisation of measurement operations by the IGUS, the rate of change of the average value of this information operator gives an estimate of the loss of structural information of physical systems. It is a positive quantity that decreases monotonically until the equilibrium state is reached. It can thus play the role of a thermodynamic potential (section V.3). The informational representation of phenomena that underlies the construction of IBU models leads to the idea that the asymmetry of phenomena in nature can be regarded as a mere consequence of the subject's activity of measuring.

\section{What is " information?"}

The notion of "information" has to be considered in its two complementary aspects. The first one, which is almost exclusively used in physics, is that of information-knowledge and characterises the amount of information which can be acquired by the reception of a message or, more generally, by the observation of the occurrence of an event. Shannon's statistical information gives a measure of the missing information-knowledge relative to an ensemble $\mathrm{E}$ of $n$ possible events $e_{i}$ which can occur with probabilities $p_{i}$ (Shannon and Weaver 1949):

$$
\mathrm{S}(\mathrm{E})=-\mathrm{k} \quad \sum_{\mathrm{i}=1, \mathrm{n}} \quad \mathrm{p}_{\mathrm{i}} \log \mathrm{p}_{\mathrm{i}}
$$


where $\mathrm{k}$ is a multiplicative constant which is generally taken equal to 1 within the framework of communication theory. The quantum analogue of Shannon's statistical information for a quantum state D (density operator) is the von Neumann "entropy" defined as:

$$
\mathrm{S}(\mathrm{D})=-\mathrm{k}_{\mathrm{B}} \operatorname{Tr} \mathrm{D} \ln \mathrm{D}=-\mathrm{k}_{\mathrm{B}} \quad \Sigma_{\mathrm{i}=1, \mathrm{n}} \quad \beta_{\mathrm{i}} \log \beta_{\mathrm{i}},
$$

where $\mathrm{k}_{\mathrm{B}}$ is the Boltzmann constant and $\beta_{\mathrm{i}}$ the eigenvalues of $\mathrm{D}$. According to this definition, $\mathrm{S}(\mathrm{D})$ refers to a statistical ensemble of events (associated with the probabilities $\beta_{\mathrm{i}}$ ) defined by the observation of the possible outcomes of non-reductive measurements, that is, of measurements performed on observables that commute with D [Balian 1982, chap. 3]. Consequently, S(D) is obviously observer-related: it refers to "accessible" information that could be obtained by specific measurements and cannot be regarded as a quantity that characterizes the intrinsic structure of the system under consideration.

The second aspect of the notion of information we need (an aspect which original idea can indeed be traced back to Aristotle) is that of organisational or structural information. This aspect of information is generally left out in most of the accounts of the information process underlying physical phenomena (as in thermodynamics, with the subjectivist interpretation of the notion of "entropy", or even in the area of quantum information theory). Unlike information-knowledge structural information is supposed to characterise the properties that can be intrinsically assigned to a physical object, as its form or its composition -even if these properties cannot be observed.

The concept of algorithmic information that has been proposed by Chaitin and Kolmogorov (Chaitin 1977) to measure the complexity of a binary series can be used as a satisfactory measure of the information-structure inherent to a single object. Actually, the algorithmic information content of an object is not at all reliant on the possibility of defining a collection of objects through some statistical distribution and does not a priori refer to the information that could be acquired by an observation. 
The algorithmic information content (or algorithmic complexity) of a binary string of symbols is defined as the length, in bits of information, of the shortest program that produces this string as its output ${ }^{2}$. The algorithmic information content of a string gives an estimate of the irreducible information content of the string that characterizes its complexity. For example, the algorithmic information content of a regular string, such as '101010101010', which can be simply produced with the program "print six ' 10 ' 's ", is lower than the algorithmic information content of the less regular string of the same length ' 100110100011 '.

Consequently, the notion of algorithmic complexity can be used to provide a quantitative estimation of the amount of information encoded in the state of a physical object, inasmuch as such a "state" can be described, as in classical physics, by well-defined spatio-temporal configurations which can then be encoded by series of binary digits (for example, the state of a material point in classical mechanics is given by its position and its momentum which can be encoded as binary series). However, the application of the notion of algorithmic complexity to quantum physics is problematic since, as shown by many authors (d'Espagnat 1994, section 4.3) (Bitbol 1996), the quantum "state" of a physical system is not a mere "description" of a spatio-temporal reality that would pre-exist to the measurements that can be performed on this system. It cannot be merely considered as a description of properties defined at any moment but rather as a predictive tool relative to some experimental situation. Nevertheless, some authors (Zurek 1989) (Caves 1990) (Vitányi 2001) (Gács P. (2001) (Mora and Briegel 2005) have proposed to apply the notion of algorithmic complexity to quantum states according to two main approaches:

2 Note that the algorithmic information content of a string is defined to within a constant depending (only) on the choice of the universal Turing machine (or computer) used to produce this string and not on its length. Thus, the algorithmic information content of a string is unambiguously defined for a given computer. 
Mora and Briegel (2005) have proposed a concept of preparation complexity which characterizes "how difficult" it is to prepare a quantum state. These authors have proposed to define the algorithmic complexity of a quantum state as the classical algorithmic complexity of its preparation process, which can always be described as a finite sequence of quantum gates -which express simple quantum unitary operations, like for example, the Hadamard gate or the CNOT gate (see (Nielsen 1998) for a review). To be precise, according to Mora and Briegel (p. 9), the algorithmic complexity $\mathrm{k}[|\psi\rangle]$ of the pure quantum state $|\psi\rangle$ relative to a given code $\Omega$ and a gate basis B with precision parameter $\varepsilon$, is the minimal value, on all possible preparations of $|\psi\rangle$ (or "circuits") $\mathrm{C}_{\varepsilon}^{\mathrm{B}}$ built with gates from B that prepare $|\psi\rangle$ with precision $\varepsilon$, of the classical algorithmic complexity of the series of symbols $\omega^{\Omega}\left(\mathrm{C}^{\mathrm{B}}{ }_{\varepsilon}(\mid \psi>)\right)$ that encode the circuit $\mathrm{C}_{\varepsilon}^{\mathrm{B}}$ :

$$
\mathrm{k}[\mid \psi>]=_{\mathrm{df}} \min \left(\mathrm{C}_{\varepsilon}^{\mathrm{B}}\right) \quad \mathrm{k}\left[\omega^{\Omega}\left(\mathrm{C}_{\varepsilon}^{\mathrm{B}}(\mid \psi>)\right)\right]
$$

where the classical algorithmic complexity $\mathrm{k}\left[\omega^{\Omega}\left(\mathrm{C}^{\mathrm{B}}{ }_{\varepsilon}(\mid \psi>)\right)\right]$ is defined as above (that is, as the length of the smallest program, in classical bits, capable of producing $\omega^{\Omega}\left(\mathrm{C}_{\varepsilon}^{\mathrm{B}}(|\psi\rangle)\right)$.

This notion of preparation complexity has been extended by Mora and Briegel (2008, p. 4) for a mixture of pure states, noted as D below, by taking the (geometric) average preparation complexity of the pure states that appear in its "optimal" mixture obtained by minimizing over all families of pure states $\left\{\left|\psi_{\alpha}\right\rangle\right\}$ such that $D=\Sigma_{\alpha} q_{\alpha}\left|\psi_{\alpha}\right\rangle\left\langle\psi_{\alpha}\right|$.

Beside Mora and Briegel's concept of preparation complexity, it is possible to adopt a concept of encoding complexity which is closer to the classical, original Chaitin's concept. For this, one must try to give a sense to the classical definition of complexity applied to quantum states: the algorithmic complexity of a quantum state would be the length of the shortest program capable of describing this state (Zurek 1989) (Caves 1990) (Vitányi 2001). It is the way a quantum state is "described" or "specified" which must be clarified in the 
quantum case. This "description" should not refer to a statistical ensemble of possible states ${ }^{3}$ in which this state is selected but to a program capable of computing the mathematical representation of this state which can be regarded, like in the classical case, as a series of symbols -and in this case the question of the computability of this state arises. Accordingly, Vitányi (p. 5) has proposed to define the algorithmic complexity of the quantum state $\mid x>$ with respect to the quantum ${ }^{4}$ Turing machine $\mathrm{Q}$ and with $\mathrm{y}$ as conditional input as the length, in classical bits, of the smallest program $\mathrm{p}$ capable of computing the state $|z\rangle$, which is the computable part of $|x\rangle$, taking into account the fidelity of the computation measured by the quantity $|\langle x \mid z\rangle|^{2}$ :

$$
\mathrm{k}_{\mathrm{Q}}[\mid \mathrm{x}>/ \mathrm{y}]==_{\mathrm{df}} \min _{\mathrm{p}}\left\{1(\mathrm{p})+\left\lceil-\log \left|\left\langle\mathrm{x}|\mathrm{z}>|^{2}\right\rceil: \mathrm{Q}(\mathrm{p}, \mathrm{y})=\right| \mathrm{z}>\right\},\right.
$$

where $1(p)$ is the length, in classical bits, of the program $p$ and $\left\lceil-\log |<x| z>\left.\right|^{2}\right\rceil$ is the length of the code word corresponding to the quantity $|\langle x \mid z\rangle|^{2}$ in the optimal, "Shannon-Fano" code associated to the probability distribution defined by $\mathrm{P}(\mathrm{x})=|\langle\mathrm{x} \mid \mathrm{z}\rangle|^{2}$.

Like for the notion of preparation complexity, that of encoding complexity can be extended for a mixture: the encoding complexity of a density operator D can be defined as the average encoding complexity of the pure states that appear in its "optimal" mixture obtained by minimizing over all families of pure states $\left\{\left|\psi_{\alpha}\right\rangle\right\}$ such that $D=\Sigma_{\alpha} q_{\alpha}\left|\psi_{\alpha}\right\rangle\left\langle\psi_{\alpha}\right|$. An important property of this encoding notion of complexity is that for a given Turing machine (a computer) and a conditional input, the complexity of a density operator increases with the number of pure states $\mid \psi_{\alpha}>$ that must be used for its description since computing a bigger number of pure states requires a longer program. In particular, in a reduction process in which

3 Which seems to be the case in the example given by Caves where he refers to the microcanonical ensemble (Caves 1990, p. 104). 
the state $\mathrm{D}$ of a physical system is projected onto the sub-space relative to the outcome $\mathrm{a}_{\alpha}$ of the measurement of some observable A:

$$
\mathrm{D} \rightarrow \mathrm{D}^{\prime}=\mathrm{P}_{\alpha} \mathrm{DP}_{\alpha} / \operatorname{Tr} \mathrm{D} \mathrm{P}_{\alpha}
$$

where $P_{\alpha}$ is the eigenprojector of $A$ associated with the outcome (eigenvalue of $A$ ) $a_{\alpha}$, the encoding complexity of the reduced state $\mathrm{D}$ ' is smaller than that of the initial state D:

$$
\mathrm{k}\left(\mathrm{D}^{\prime}\right)<\mathrm{k}(\mathrm{D})
$$

since in the eigenbasis of A (where D' can be optimally described) all the terms of D relative to other possible outcomes have been rubbed out in this process. This property will be used in the following sections.

These two notions of algorithmic complexity of the quantum state that can be associated with a physical system, which focus either on the difficulty for preparing this state or on the amount of information needed to compute it, can be used to characterize its organizational or structural information. An "information" which is not reducible to that of informationknowledge about the possible outcomes of specific measurements that can be performed on this system. If the concept of information-knowledge is powerful in order to deal with the acquisition of "information" by the subject of knowledge and action (in section II), the informational analysis of the evolution of physical systems (in section V) indeed requires to appeal to the concept of information-structure, which focus on the intrinsic organization of the "object" under consideration and not on what can be known about it by an observation (and even if its definition is computer-dependent, base-dependent or use a notion of fidelity of

4 Indeed, the notion of a quantum computer is not essential since its work can always be simulated by a classical computer provided the question of the computation time is not taken into account (Vitányi 2001, p. 5) (Gács P. 2001). 
the computation, which introduces some element of "subjectivity"). Both notions of complexity of a quantum state will be used in this paper.

\section{The informational branching universe.}

As explained in the introduction, the construction of the evolving models of the informational universe makes use of Everett's original formulation of the measurement operation (the "relative state" formulation) for which no ontological ("many worlds") interpretation is given. The histories of IBU models are not actual histories of co-existing physical worlds but they are the histories of the possible informational states of the IGUS, only one of them being actualised. Let us now precise the construction of such models.

The elementary acts of construction of the informational universe, the elementary "events", are defined by the acquisition of one bit of information by some IGUS in a measurement operation. The notion of IGUS (information gathering and utilizing system) has been suggested by Gell-Mann and Hartle (1990) in their cosmological theory and refers to any "complex adaptive system that has evolved to exploit the relative predictability of a quasiclassical domain" (p. 426). However, in this theory the emergence of the quasiclassical domain is based on a concept of coarse graining which is not necessary here.

The acquisition of information-knowledge by an IGUS in a measurement operation, which defines an informational "event", is correlative to the reduction of the initial state. When an ideal measurement of the observable $\mathrm{M}$ is performed on a quantum system $\mathrm{S}$ by an apparatus A, a pure state can be associated to the joint system S-A just after their interaction. This pure state is rapidly transformed into a mixture of states corresponding to the eigenvalues of the observable that has been measured, only one of them being actually observed. This reduction process can be written in the formalism of density operators (and using Dirac's notation) as follows : 
$\mathrm{D}_{\text {in }}=\Sigma_{\mathrm{m}, \mathrm{n}} \mathrm{a}_{\mathrm{m}} \mathrm{a}_{\mathrm{n}} *\left|\psi_{\mathrm{m}}>\right| \phi_{\mathrm{m}}><\psi_{\mathrm{n}}\left|<\phi_{\mathrm{n}}\right|$

$$
\rightarrow \mathrm{D}_{\mathrm{fin}}=\Sigma_{\mathrm{m}}\left|\mathrm{a}_{\mathrm{m}}\right|^{2}\left|\psi_{\mathrm{m}}>\right| \phi_{\mathrm{m}}><\psi_{\mathrm{m}}\left|<\phi_{\mathrm{m}}\right|
$$

where $\left|\psi_{\mathrm{m}}\right\rangle$ are the eigenstates of the measured observable $M$ and $\left|\phi_{\mathrm{m}}\right\rangle$ are the corresponding "pointer states" of the measurement apparatus A. $\mathrm{D}_{\text {in }}$ is the pure state that can be associated to the system S-A just after their interaction and $\mathrm{D}_{\text {fin }}$ is the mixture that describes the statistical distribution of the possible pointer states.

In this transition, the "crossed" terms of the form $\left|\psi_{\mathrm{m}}>\right| \phi_{\mathrm{m}}><\psi_{\mathrm{n}}\left|<\phi_{\mathrm{n}}\right|$ for different $\mathrm{m}$ and $\mathrm{n}$ have been rubbed out. Consequently, if, from the observer's point of view, the von Neumann "entropy" of the final state, which evaluates the missing information-knowledge relative to subsequent measurements that could be performed on $\mathrm{S}$, is higher than that of the initial state (Balian 1982, p. 105):

$$
\Delta \mathrm{S}=\mathrm{S}\left(\mathrm{D}_{\mathrm{fin}}\right)-\mathrm{S}\left(\mathrm{D}_{\mathrm{in}}\right)>0,
$$

its encoding complexity, which gives an evaluation of its information content, is smaller than that of $\mathrm{D}_{\text {in }}$ (see section I):

$$
\mathrm{k}\left[\mathrm{D}_{\text {fin }}\right]<\mathrm{k}\left[\mathrm{D}_{\text {in }}\right] .
$$

This selective loss of information-structure in a measurement is due to the decoherence process which has been explored in detail by Zurek (2003). Decoherence is caused by the interaction of the measuring device (which is an open system) with the environment which monitors some of its observables. Indeed, the environment-induced superselection first leads to the resolution of the question of the basis ambiguity -that is, the question to know which observable is really measured by the device. For the Hamiltonian of interaction between the apparatus and the environment "chooses" the observable of the apparatus that can be measured without destroying the information it has acquired about the measured system. Second, it leads to the very rapid transformation of the initial pure state associated with the composed system S-A just after their interaction into a stable mixture of the "einselected" 
states. That is, entanglement between $\mathrm{A}$ and E rapidly turn into classical correlations as a result of the interaction between $\mathrm{A}$ and its environment. Classicality then appears as a consequence of the decoherence process. Moreover, Zurek has shown that Born's rule, which permits the calculation of predictions, can be justified from the environment-assisted invariance, and without any appeal of a concept of coarse-graining, by considering what can (or cannot) be known about the system entangled with its environment. Given the joint state of the system and the environment, Born's probabilities appear as the only ones that can be assigned to the observation of the possible pointer states under the condition of environmentassisted invariance (Zurek 2003).

The phenomenon of decoherence is in fact essential to give a definite meaning to the branching dynamics which relies on the separation and the stability of the different possible outcomes of the measurement, only one of them being actually observed by the IGUS. As explained in the introduction, it is the selection of a sequence of information among all the possible ones that constitutes the actual history of the informational universe.

One will consider that the IGUS is endowed with a memory (which is assumed erasureresistant, for simplification) where information relative to measurements can be recorded and that he is capable of performing some operations in some specific context whose description can be incorporated in the memory content. The IGUS must indeed be understood as an intersubjective entity since the subject of experience and action (the "Observer") is not considered for his empirical characteristics but for his ability to gather information by acting in the world, an ability which is shared by all empirical subjects (as it happens, by the community of physicists).

The wave function of the universe can be written as a product of the wave functions that can be associated with its constituents, where the state of the IGUS is endowed with a memory and a set of possible actions (extending Everett's notations): 


$$
\psi=\psi^{\mathrm{S} 1} \psi^{\mathrm{S} 2} \quad \ldots \ldots \psi^{\mathrm{IGUS}}{ }_{[\mathrm{memory}} ; \text { actions] } .
$$

If the IGUS measures on the system S1 the observable A with eigenfunctions $\phi^{\mathrm{S} 1}{ }_{\alpha}$ the initial wave function:

$$
\psi=\psi^{\mathrm{S} 1} \psi^{\mathrm{S} 2} \quad \ldots \ldots \psi^{\mathrm{IGUS}}[\ldots \ldots ; \text { actions }]
$$

where the IGUS's memory is empty transforms into:

$$
\psi_{\alpha}=\Sigma_{\alpha} \mathrm{a}_{\alpha} \phi^{\mathrm{S} 1}{ }_{\alpha} \psi^{\mathrm{S} 2} \ldots \ldots . . \psi^{\text {IGUS }}{ }_{\alpha\left[\ldots, \mathrm{i}_{\alpha} ; \text { actions }\right]},
$$

where $\mathrm{a}_{\alpha}=\left\langle\phi^{\mathrm{S} 1_{\alpha}} \mid \psi^{\mathrm{S} 1}\right\rangle$ and $\mathrm{i}_{\alpha}$ is the information-knowledge corresponding to the eigenfunction $\mid \phi^{S 1}{ }_{\alpha}>$ which is recorded in the IGUS's memory.

When the IGUS records $i_{\alpha}$ he is said to be in the "relative state" :

$$
\psi \text { IGUS }_{\alpha\left[\ldots, \mathrm{i}_{\alpha}, \text { actions }\right]} .
$$

According to quantum theory, the transformations $\psi \rightarrow \psi_{\alpha}$ are weighted according to Born's rule :

$$
\mu\left(\psi \rightarrow \psi_{\alpha}\right)=\left|\mathrm{a}_{\alpha}\right|^{2}
$$

The construction of the informational branching model relies on the process of acquisition of information-knowledge by the IGUS. Thus, in his section, we will exclusively focus on his memory content and the set of the possible actions that he can perform in each of the possible "relative states", considering the rest of the decomposition of secondary importance":

$$
\psi=\psi^{\mathrm{S} 1} \psi^{\mathrm{S} 2} \ldots \ldots . . . \psi^{\mathrm{IGUS}}[\text { [memory } ; \text { actions] }
$$

A memory state of the IGUS, noted as $\mathrm{E}_{\alpha}$, will thus be understood as the doublet:

$$
\mathrm{E}_{\alpha}=\left(\mathrm{D}_{\alpha}, \mathrm{O}_{\alpha}\right)
$$

where $\mathrm{O}_{\alpha}$ is the set of operations that can be performed in the state $\mathrm{E}_{\alpha}$ and $\mathrm{D}_{\alpha}$ is the information-knowledge relative to the experimental data recorded in its memory. The IGUS's

5 On the contrary, in section $\mathrm{V}$ we will focus on the "object"-part of the decomposition of the universal wave function. 
activity takes place in a theoretico-experimental context (namely, the quantum paradigm) which is supposed not to change. Moreover, unlike the set of data $\mathrm{D}_{\alpha}$, it is also assumed, in a first approximation, that the set of operations $\mathrm{O}_{\alpha}$ does not change when new information is recorded by the memory. In conformity with Everett's notation, $\mathrm{D}_{\alpha}$ is written as a sequence in brackets $\left[\ldots, i_{\beta}, \ldots, i_{\alpha}\right]$ where $i_{\alpha}$ is the "last" information recorded in the memory (the question of the presupposed linear time frame will be explored below.)

A strict partial ordering relation on the set of all possible memory states of the IGUS can be defined as follows :

$E_{\alpha} \mathbf{R} E_{\alpha}$ if $D_{\alpha}$, is a strict final extension of $D_{\alpha}$.

As shown by this definition, and contrary to the objectivist interpretation of the ordering relation in BST models mentioned in the introduction, it is the process of information acquisition (and not the relativistic causal relation) that defines the partial ordering relation on the IGUS's memory states (and not on a set of abstract point-events).

The ordered set of memory states is discrete since the series $\mathrm{D}_{\alpha}$ can only be extended by addition (at right) of discrete unities: the symbols of the language which is used to encode the data. Moreover, it is easy to see that $\mathbf{R}$ satisfies the property of linearity of the past (or tree property), which means that the set of predecessors (by the relation $\mathbf{R}$ ) of any informational sate of the IGUS is totally ordered. Moreover, it is assumed that $\mathbf{R}$ satisfies the property of connectedness according to which any couple of memory states of the IGUS has a greatest lower bound according to $\mathbf{R}$, which is the formal expression of the idea of unicity of the informational universe. The informational branching model of the universe are thus evolving structures whose steps of construction can be indexed by a "time" parameter $\tau$ (its definition will be clarified in the next section) :

$$
\mathbf{U}(\tau)=\langle\mathrm{E}, \mathbf{R}\rangle,
$$


where $E=\left\{E_{\alpha}\right\}$ is the discrete set of memory states of the IGUS and $\mathbf{R}$ is the strict, partial, connected ordering relation defined above. Indeed, IBU models satisfy the axioms of the discrete branching time models (Burgess 1978) (Prior 1967) but the "instants" of time are here interpreted by the possible IGUS's memory states (see Appendix). Note that, as briefly mentioned in the introduction, the concept of indeterminism of the future (memory states) in IBU models is that of antactualist (or Piercean) branching time logic: it is not thought as the expression of our ignorance but as a fundamental feature of becoming. Moreover, in spite of the fact that, like BST models, they use the set-theoretical methods for their definition, they are not given once and for all, from an external point of view, but they are the very expression of the universal process of information due to the IGUS's activity of measuring. They do not result from an abstract pasting of space-time manifolds but they are evolving, informational branching structure which are progressively constructed from the realization of measurement operations.

\section{Histories and Probabilities.}

The possible histories of the informational universe are maximal sets of memory states totally ordered by the relation $\mathbf{R}$. Let us remind that, according to our epistemic view on quantum indeterminism, only one of these possible histories, the actual one (even if unknown), is in fact realized.

In section II, one has mentioned the fact that, in a non-branching universe, the loss of information-structure due to the decoherence phenomenon allows the emergence of noninterfering and stable classical states to which probabilities can be associated according to Born's rule. However, can probabilities be assigned to alternative histories built up with such decoherent states in a branching universe ? To be precise, are histories of IBU models (and, in fact, those of any branching universe) "consistent" histories in the sense of Griffiths (1984), 
that is, can these histories be still considered as sequences of classical events ruled by the classical probability rules?

In Griffith's notation, a history $h_{j}$ relative to a temporal sequence of "real" properties of the physical universe can be written as a time-ordered sequence of operators in the Heisenberg picture:

$$
h_{j}=\left(P_{\alpha_{1}}^{1}\left(t_{1}\right), P^{2} \alpha_{2}\left(t_{2}\right), \ldots ., P^{k}{ }_{\alpha k}\left(t_{k}\right), \ldots, P^{n} \alpha_{n}\left(t_{n}\right)\right)
$$

where the index $\alpha_{k}$ denotes the particular event that occurs at time $t_{k}$ in the set, indexed by $k$, of alternative projectors. However, from our epistemic point of view, in IBU models, each projector corresponds to some information which can be recorded in the IGUS's memory. In other words, a history of the informational universe should, in fact, be written as the sequence of the corresponding IGUS's memory states:

$$
h_{j}=\left(E\left(t_{1}\right), \ldots, E\left(t_{k}\right), \ldots, E\left(t_{n}\right)\right)
$$

where $\mathrm{E}\left(\mathrm{t}_{\mathrm{k}}\right)=\left(\mathrm{D}\left(\mathrm{t}_{\mathrm{k}}\right), \mathrm{O}\left(\mathrm{t}_{\mathrm{k}}\right)\right.$ ) and where $\mathrm{D}\left(\mathrm{t}_{\mathrm{k}}\right)$ contains, among a multitude of other information, the information relative to the observation of the sequence $\left(\mathrm{P}^{1} \alpha_{1}\left(\mathrm{t}_{1}\right), \mathrm{P}^{2}{ }_{\alpha_{2}}\left(\mathrm{t}_{2}\right)\right.$, $\left.\ldots ., \mathrm{P}^{\mathrm{k}}{ }_{\alpha_{\mathrm{k}}}\left(\mathrm{t}_{\mathrm{k}}\right)\right)$. Nevertheless, for simplicity of writing, one will adopt Griffith's notation that focuses on the properties that can be assigned to physical systems and not on the sequence of the corresponding IGUS's memory states. Accordingly, in order to focus on the evolution of a single physical system S, it will be more convenient to work in an appropriate sub-structure of $\mathbf{U}_{S}$ of $\mathbf{U}$ where the informational content of the IGUS's memory is reduced to the only information relative to $S$ (sections $\mathrm{V}$ and $\mathrm{VI}$ ).

In order to define a classical probability distribution to such sets of alternative histories we would like to apply Wigner's formula, which generalises Born's rule in the case of a series of measurements that are performed successively in a linear time frame: 


$$
\mu\left(h_{j}\right)=\operatorname{Tr}\left(P_{\alpha_{n}}^{n}\left(t_{n}\right) \ldots \ldots P^{1}{ }_{\alpha_{1}}\left(t_{1}\right) \rho_{H} P^{1}{ }_{\alpha_{1}}\left(t_{1}\right) \ldots . . P^{n} \alpha_{n}\left(t_{n}\right)\right)
$$

$\rho_{\mathrm{H}}$ being the density operator that represents the state of the (physical) universe in the Heisenberg picture.

However, in the case of a branching universe how is defined the time parameter involved in these formula ? Obviously, this calculation can be applied to alternative histories of a branching universe only in the case these histories can be referred to the same linear time frame where the values $t_{\mathrm{k}}$ of all these alternative histories are defined. If not, the quantities $\mu\left(h_{j}\right)$ for the alternative histories $h_{j}$ do not define at all a probability distribution (these histories cannot even be considered as "alternative" since there cannot be compared according to the same linear time scale !) In spite on its unquestioned use by some physicists (Omnès 2001)(Gell-Mann and Hartle 1990), the possibility of assigning a meaning to Wigner's formula (or, equivalently, to Feynman's sum-over-histories formulation of quantum mechanics) in a branching universe is conditioned to the possibility of defining an only linear time frame to which the decohering alternative histories of the universe can be referred.

Unless assuming that the splitting of histories always occur along very special, equal-time hyperplanes, this question seems to require the definition of an universal linear time frame to which the alternative decohering histories can be referred -which seems to give rise to a serious difficulty. Fortunately, such a "synchronization" of constantly evolving histories can be achieved in the models of the informational branching universe. A universal "time" parameter can be defined as a measure of the informational content of the memory states of the IGUS. This time scale is defined according to the usual propositions to foliate relativistic spacetime in hypersurfaces labeled by a specific parameter that can play the role of "intrinsic time" for the universe (for example, a parameter relying on its expansion (Zeh 1989, chap 6)). However, in the present proposal, the foliation parameter is not defined in physical terms but in computational ones, as a measure of the IGUS's memory content. This informational 
"time" scale, which should not be confused with the external, absolute Newtonian time defined independently of any phenomenon (and any observer), constitutes nevertheless a strict total order to which all the alternative histories of a branching universe can be referred.

To be precise, the information content of a state $\mathrm{E}_{\alpha}$ of the IGUS's memory can be defined as the algorithmic information content $\mathrm{k}\left(\mathrm{D}_{\alpha}\right)$ of the sequence $\mathrm{D}_{\alpha}$, making abstraction of the constant sets $\mathrm{O}_{\alpha}$ and $\mathrm{C}$. The quantity $\mathrm{k}\left(\mathrm{D}_{\alpha}\right)$ can play the role of a universal "time" parameter defined from the models of branching informational universe (and not from an external point of view). Indeed, for any (arbitrary) strictly increasing function f defined from the set of natural numbers to the set of real numbers a "time"-information parameter $\tau_{\alpha}$ that evaluates the algorithmic informational content $\mathrm{I}\left(\mathrm{E}_{\alpha}\right)$ of the sate $\mathrm{E}_{\alpha}$ can be defined:

$$
\tau_{\alpha}=\mathrm{I}\left(\mathrm{E}_{\alpha}\right)=\mathrm{f}\left(\mathrm{k}\left(\mathrm{D}_{\alpha}\right)\right)
$$

The structure $\mathrm{T}=\left(\left\{\tau_{\alpha}\right\},<\right)$, where the order relation " $<$ " is the usual total order on real numbers (restricted to $\operatorname{Im} \mathrm{f}$ ), is a strict, discrete, total order to which the alternative histories of the informational universe can be referred. Moreover, it can be used to index the steps of construction of IBU models (like in the previous section).

The possible histories of the informational universe can be said "synchronized" by the possibility of defining such an informational "time" scale in which the sequences of classical events that define the possible histories take place. This allows the application of Wigner's formula in IBU models :

$$
\mu_{j}={ }_{d f} \mu\left(h_{j}\right)=\operatorname{Tr}\left(P_{\alpha_{n}}^{n}\left(\tau_{n}\right) \ldots \ldots . P^{1}{ }_{\alpha_{1}}\left(\tau_{1}\right) \rho_{H} P_{\alpha_{1}}^{1}\left(\tau_{1}\right) \ldots \ldots P_{\alpha_{n}}^{n}\left(\tau_{n}\right)\right)
$$

where the "time" parameter $\tau$ ranges on the only linear informational scale T.

According to this proposal, the above probability $\mu_{\mathrm{j}}$ refers to the history defined by the successive values of the universal informational parameter $\tau$ and not by those of a particular time parameter defined for some specific relativistic universe. To be precise, a projector 
$\mathrm{P}^{\mathrm{i}}{ }_{\alpha_{\mathrm{i}}}\left(\tau_{\mathrm{i}}\right)$ now refers to the measurement event indexed by $\alpha_{\mathrm{i}}$ for the specific value $\tau_{\mathrm{i}}$ of $\tau$, that is, for this particular size of the IGUS's memory -a specific value of $\tau$ which could be related (by means of appropriate functions) to the spatial coordinates and the proper time of any observer of the hypersurface labeled by this value $\tau_{\mathrm{i}}$ of $\tau$. The applicability of (generalized) Born's rule for a sequence of measurements does not presuppose that this succession takes place along a particular time scale (Newtonian, for example). Its application only requires to be given the state $\rho_{\mathrm{H}}$ of the system and the eigenprojectors associated with the series of outcomes of measurement under consideration, independently of the particular time scale these projectors (or this state) can be referred to. Here, the probability $\mu_{\mathrm{j}}$ defined above must be understood as the probability relative to the series of measurement-events indexed by the ordered series of values $\tau_{\mathrm{i}}$ of the "time"-information parameter running on the total order T defined above.

As explained in section II, the realization of measurement operations by the IGUS gives rise to the recording of some (corresponding) information-knowledge in its memory. Consequently, the increase of the IGUS' s memory content can define the "flow" of timeinformation, which measure is given by the parameter $\tau$ defined above. In other words, in the proposed model, the "temporal" asymmetry is the expression of the information process relative to the realization of measurement operations by the IGUS. The flow of timeinformation cannot thus be considered as a strongly objective feature of the external world since the IGUS's actions and observations are involved in its definition.

The informational models of the universe are thus defined as branching structures weighted by the probability distribution $\mu$ and synchronised by the function $\mathrm{k}$ which evaluates the information content of the IGUS's memory states :

$$
\mathbf{U}(\tau)=\langle\mathrm{E}, \mathbf{R}, \mu, \mathrm{k}\rangle
$$




\section{The representation of physical states in the informational branching universe.}

In order to deal with a particular physical system $S$ it is more convenient to consider substructures

$$
\mathbf{U}_{\mathrm{S}}(\tau)=\left\langle\mathrm{E}_{\mathrm{S}}, \mathbf{R}_{\mathrm{S}}, \mu_{\mathrm{S}}, \mathrm{k}_{\mathrm{S}}>\right.
$$

of $\mathbf{U}(\tau)$, where $E$ is reduced to the set $\mathrm{E}_{\mathbf{S}}$ of informational states of the IGUS relative to $S$. $E_{S}$ is ordered by the restriction $\mathbf{R}_{S}$ of $\mathbf{R}$ on $E_{S}, k_{S}$ is the restriction of $k$ on $E_{S}$ and $\mu_{S}$ weights the histories constituted by sequences of memory states of $E_{S}$. Note that focusing on the sub-structure $\mathbf{U}_{\mathrm{S}}(\tau)$ should not make us forget the fact that the "system" $\mathrm{S}$ is a part of the whole physical universe which is submitted to the universal branching dynamics triggered by the realization of any measurement operation by the IGUS (see section II). In other words, the branching of histories in $\mathbf{U}_{\mathrm{S}}(\tau)$ is not only the consequence of local measurement operations performed on S but, even if S can be considered as "isolated", it can be triggered by any measurement operation performed on any part of the physical universe by the IGUS.

The informational branching model $\mathbf{U}_{\mathrm{S}}(\tau)$ associated to the evolution of the physical system S naturally allows a historical representation of its state. That is, a formal representation that encodes, at each "time" $\tau$ of the informational linear scale $\mathrm{T}$, all the information about all its possible evolutions along the ("growing") branches of the substructure $\mathbf{U}_{\mathrm{S}}(\tau)$. This historical representation is made possible within an extended representation space defined as follows.

The historical quantum state associated with a physical system $\mathrm{S}$ for some value $\tau$ of the time-information parameter is a vector of the following space:

$$
\mathrm{H}(\tau)={ }_{\mathrm{df}} \oplus_{\mathrm{B}(\tau)} \quad \mathcal{H}_{\mathrm{j}}
$$


$H(\tau)$ is the direct sum, on the set $B(\tau)$ of all branches of $\mathbf{U}_{S}(\tau)$, of copies :

$$
\mathcal{H}_{\mathrm{j}}=\mathrm{df} \mathcal{H} \times\left\{b_{\mathrm{j}}\right\}
$$

indexed by the branch $\mathrm{j}$, of the Hilbert space $\mathcal{H}$ on complex numbers defined as usually for $\mathrm{S}$.

For practical reasons the number of branches that are created at a splitting point can be considered as finite. For any measurement has a finite resolution and the set of values that can actually be measured by a measuring device is always bounded (even if the spectrum of possible values predicted by quantum theory is continuous and ranges over the set of real numbers.)

The space $H(\tau)$, which is the orthogonal sum of a finite number of orthogonal Hilbert spaces $\mathcal{H}_{\mathrm{j}}$, is an Hilbert space. To be precise, the extended space $H(\tau)$ is constructed as follows:

1) One first considers the isomorphic copies $\mathcal{H}_{\mathrm{j}}$ of the Hilbert space $\mathcal{H}$ defined as usually for a physical system. On these copies can be defined the notions of sum, product by a complex number, hermitian scalar product and norm by extending the similar notions in $\mathcal{H}$ (the vectors of $\mathcal{H}_{\mathrm{j}}$ are noted according to Dirac's notations, or, more generally, as vectors of $\mathcal{H}$ with the exponent $\mathrm{j})$ :

$$
\begin{gathered}
\left|\psi^{j_{1}}\right\rangle+\left|\psi^{j_{2}}\right\rangle=d_{d f}\left(\left|\psi_{1}\right\rangle+\left|\psi_{2}\right\rangle\right)^{j} \\
\beta\left|\psi^{j}\right\rangle={ }_{d f}(|\beta \psi\rangle)^{j} \\
\left\langle\psi^{j}{ }_{1} \mid \psi^{j_{2}}\right\rangle={ }_{d f}\left\langle\psi_{1} \mid \psi_{2}\right\rangle=\left\langle\psi_{2} \mid \psi_{1}\right\rangle *=\left\langle\psi^{j}{ }_{2} \mid \psi^{j}{ }_{1}\right\rangle * \\
\|\left|\psi^{j}\right\rangle\left\|=_{\text {df }}\right\||\psi\rangle \|=(\langle\psi \mid \psi\rangle)^{1 / 2}
\end{gathered}
$$

A vector of $H(\tau)$ can be written as $V=\left(\left|\psi^{1}{ }_{\alpha}\right\rangle, \ldots .,\left|\psi^{\mathrm{n}} \gamma\right\rangle\right)$, where $\mathrm{n}$ is the (finite) number of branches that are created at $\tau$ and $\left|\psi^{1}{ }_{\alpha}\right\rangle, \ldots .,\left|\psi^{\mathrm{n}} \gamma\right\rangle$ are the vectors of the orthogonal sub-spaces $\mathcal{H}_{\mathrm{j}}$ respectively obtained from the vectors $\left|\psi_{\alpha}\right\rangle, \ldots .,\left|\psi_{\gamma}\right\rangle$ of $\mathcal{H}$. 
2) One then defines the notions of sum, product by a complex number, hermitian scalar product and norm for the vectors of the generalized spaces $H(\tau)$ - for simplicity of writing, one will note a vector of $H(\tau)$ by its general $\mathrm{j}$-component in the space $\mathcal{H}_{\mathrm{j}}$, that is, $\mathrm{V} \equiv(\mid \psi$ $\mathrm{j}_{\lambda>)}$

$$
\begin{gathered}
V_{1}+V_{2}=_{d f}\left(\left|\psi \lambda_{1}\right\rangle+\left|\psi \lambda_{2}\right\rangle\right)^{j} \\
\beta V==_{d f}(\beta|\psi \lambda\rangle)^{j}, \text { where } \beta \text { is a complex number } \\
V_{1} \cdot V_{2}=_{d f} \sum_{B(\tau)}\left\langle\psi^{j} \lambda_{1} \mid \psi^{j}{ }_{\lambda 2}\right\rangle=\left(V_{2} \cdot V_{1}\right)^{*} \\
\|V\|={ }_{d f}\|V . V\|^{1 / 2}
\end{gathered}
$$

When there is no need to specify the vectors $\left|\psi_{\alpha}\right\rangle, \ldots .,\left|\psi_{\gamma}\right\rangle$ of $\mathcal{H}$, the vector $\mathrm{V}$ of $H(\tau)$ which can be associated, at $\tau$, to the quantum state of $S$ can be simply written as :

$$
\left.\mathrm{V}=\left(\left|\psi^{1}>, \ldots .,\right| \psi^{\mathrm{n}}\right\rangle\right) \equiv \Sigma_{\mathrm{j}} \mathrm{V}^{\mathrm{j}}
$$

where $\mathrm{V}^{\mathrm{j}}=\left(\left|0^{1}>, \ldots .,\right| \psi^{\mathrm{j}}>, \ldots . ., \mid 0^{\mathrm{n}}>\right)$.

To underscore the weights $\mu_{\mathrm{j}}$ of the histories $\mathrm{h}_{\mathrm{j}}$, one has to use vectors of unit-norm and to write $\mathrm{V}$ as :

$$
\mathrm{V}=\left(\mathrm{a}_{1}\left|\psi^{1}>, \ldots ., \mathrm{a}_{\mathrm{n}}\right| \psi^{\mathrm{n}}>\right) \equiv \Sigma_{\mathrm{j}} \mathrm{a}_{\mathrm{j}} \mathrm{V}^{\mathrm{j}},
$$

where $\left\|\mathrm{V}^{\mathrm{j}}\right\|=\left\|\left|\psi^{\mathrm{j}}>\|=\|\right| \psi>\right\|=1$, for all $\mathrm{j}$, and where $\mu_{\mathrm{j}}=\left|\mathrm{a}_{\mathrm{j}}\right|^{2}$ is the probability associated with the history $h_{j}$ at $\tau$ according to Wigner's formula applied in $\mathbf{U}_{\mathrm{S}}$ (see section V). Note that the $a_{j}$ satisfy the normalization condition : $\Sigma_{j}\left|a_{j}\right|^{2}=1$.

The vectors $\mathrm{V}^{\mathrm{j}}$ of the space $\mathrm{H}(\tau)$, which are the j-“components" of the state vector $\mathrm{V}$ associated to $S$ at $\tau$, have a physical meaning: they result from the successive projections of the initial state vector of $S$ onto the subspaces associated with the series of "events" that defines the j-branch (see section III). Thus, all the possible (past) histories $\mathrm{h}_{\mathrm{j}}$ of $\mathrm{S}$ constituted 
by the events that occurred since some instant taken as "initial" are taken into account in the state $\mathrm{V}$ via its $\mathrm{j}$-components $\mathrm{V}^{\mathrm{j}}$. In this sense, the state vector associated to a physical system can be regarded as a "historical" state.

A notion of density operator, which generalizes that of state vector for a mixture, can also be defined in the spaces $H(\tau)$ :

$$
\mathrm{D}=\Sigma_{\mathrm{j}} \mu_{\mathrm{j}} \mathrm{D}^{\mathrm{j}}
$$

where $\mu_{j}$ are the probabilities relative to the alternative histories $b_{j}$ defined above and $D^{j}$ is the representation of the formal state of $\mathrm{S}$ in $\mathcal{H}_{\mathrm{j}}$. It can be checked that the operator $\mathrm{D}$ is hermitian and that $\operatorname{Tr} \mathrm{D}=1$, where the trace operation is defined as usually.

The density operator $\mathrm{D}$ encodes the maximal information (accessible or not by measurements) which is available at "time" $\tau$. That is, it encodes not only the (usual) uncertainty regarding the state of $S$ in the spaces $\mathcal{H}_{\mathrm{j}}$, but also the uncertainty regarding the history of $\mathbf{U}_{\mathrm{S}}$ where $\mathrm{S}$ can be located at $\tau$.

3) An operator algebra acting on the generalized spaces $H(\tau)$ can be defined in a similar way to the algebra of operators acting on the original Hilbert space $\mathcal{H}$. On this algebra are defined a linear sum, a product by a complex number, a non-commutative product and an operation of hermitian conjugation :

$$
\begin{aligned}
&\left(\mathrm{O}_{1}+\mathrm{O}_{2}\right) \mathrm{V}={ }_{\mathrm{df}} \mathrm{O}_{1} \mathrm{~V}+\mathrm{O}_{2} \mathrm{~V} \\
& \lambda \mathrm{OV}={ }_{\mathrm{df}} \mathrm{O}(\lambda \mathrm{V}) \\
& \text { commutator of } \mathrm{O}_{1} \text { and } \mathrm{O}_{2}: \quad\left[\mathrm{O}_{1}, \mathrm{O}_{2}\right]={ }_{\mathrm{df}} \mathrm{O}_{1} \mathrm{O}_{2}-\mathrm{O}_{2} \mathrm{O}_{1}
\end{aligned}
$$

hermitian conjugation : to any operator $\mathrm{O}$ acting on $\mathrm{H}(\tau)$ can be associated the operator $\mathrm{O}^{+}$ such that its action on the element $\mathrm{V}^{+}$of the dual space $\mathrm{H}^{+}(\tau)$, noted as $\mathrm{V}^{+} \mathrm{O}^{+}$, is the dual form of $\mathrm{OV}$. 
Like in $\mathcal{H}$, the functions of operators acting on $\mathrm{H}(\tau)$ are defined from complex variables functions expandable into power series.

Note that it is possible to define in $H(\tau)$ a specific but nevertheless very useful class of operators that are "extensions" of operators acting on the original Hilbert space $\mathcal{H}$. For any operator $A_{0}$ of $\mathcal{H}$, the action of its extension, called $A$, on a vector $V=\Sigma_{j} a_{j} V^{j}$ of $H(\tau)$ is defined as:

$$
\mathrm{A} V={ }_{\mathrm{df}} \quad\left(\mathrm{a}_{1} \mathrm{~A}_{0}{ }^{1}\left|\psi^{1}>, \ldots ., \mathrm{a}_{\mathrm{n}} \mathrm{A}_{0}{ }^{\mathrm{n}}\right| \psi^{\mathrm{n}}>\right) \equiv \Sigma_{\mathrm{j}} \mathrm{a}_{\mathrm{j}} \mathrm{A}_{0}{ }^{\mathrm{j}} \mathrm{V}^{\mathrm{j}}
$$

where $\mathrm{A}_{0}^{\mathrm{j}} \mid \psi^{\mathrm{j}\rangle}=_{\mathrm{df}}\left(\mathrm{A}_{0} \mid \psi>\right)^{\mathrm{j}}$. The action of such an "extended", multi-branch operator is thus distributed onto the different possible histories constructed at $\tau$. In particular, one can define the extended Hamiltonian operator $\mathrm{H}$ as :

$$
\mathrm{HV}=\Sigma_{\mathrm{j}} \mathrm{a}_{\mathrm{j}} \mathrm{H}_{0}^{\mathrm{j}} \mathrm{V}^{\mathrm{j}}
$$

where $\mathrm{H}_{0}$ is the Hamiltonian operator defined as usually in of the original space $\mathcal{H}$ and $\mathrm{H}_{0}{ }^{\mathrm{j}}$ are the corresponding operators in the spaces $\mathcal{H}_{j}$.

\section{Temporal evolution in the informational branching models of the universe.}

\section{1. Schrödinger-like evolution.}

The possibility of defining extended operators in IBU models leads to the reformulation, in $H(\tau)$, of the equations of evolution -like Schrödinger equation. Let us first calculate the “time"-information derivative, noted as $\Delta_{\tau}$, of a vector $\mathrm{V}=\Sigma_{\mathrm{j}} \mathrm{a}_{\mathrm{j}} \mathrm{V}^{\mathrm{j}} \mathrm{of} \mathrm{H}(\tau){ }^{6}$

6 This notion of "derivation" is of course an approximation of the rigorous notion of derivation where the infinitesimal change of a continuous variable is replaced by that of the smallest change of a discrete variable, namely $\tau$. However, as emphasized by Carnap (1966), the physical quantities we actually measure form discrete sets because of the finite resolution of measuring devices. Continuity of variables (which is thus always approximate in physics) is only supposed when we apply the laws that are formulated with the help of differential calculus. Moreover, note that by the definition of the informational parameter $\tau$ (section III), its change can be made as small as wanted by suitably choosing the arbitrary function $\mathrm{f}$. 
$\Delta_{\tau} \mathrm{V}=\Sigma_{\mathrm{j}} \mathrm{a}_{\mathrm{j}}(\partial / \partial \tau) \mathrm{V}^{\mathrm{j}}$

$=\Sigma_{\mathrm{j}} \mathrm{a}_{\mathrm{j}}\left(\partial \mathrm{V}^{\mathrm{j}} / \partial \mathrm{t}^{\mathrm{j}}\right)\left(\partial \mathrm{t}^{\mathrm{j}} / \partial \tau\right)$, where $\left(\partial / \partial \mathrm{t}^{\mathrm{j}}\right)$ denotes the time derivative operator in the $\mathrm{j}$-branch, acting on vectors of the $\mathcal{H}_{\mathrm{j}}$ space, and $\left(\partial \mathrm{t}^{\mathrm{j}} / \partial \tau\right)$ is the inverse of the information recording rate of the IGUS's memory along the branch $\mathrm{j}$. One can assume that this rate has the same value in all branches since the latter is a priori determined by the cognitive abilities of the IGUS and not by the space-time metric of his universe. Let this constant value be $\chi$. The time-information derivative of $\mathrm{V}$ can then be written as :

$$
\Delta_{\tau} \mathrm{V}=(1 / \chi) \Sigma_{\mathrm{j}} \mathrm{a}_{\mathrm{j}} \partial \mathrm{V}^{\mathrm{j}} / \partial \mathrm{t}^{\mathrm{j}}
$$

The evolution law can now be shown by writing the Schrödinger evolution law in each of the j-branches :

$$
i \hbar\left(\partial \mathrm{V}^{\mathrm{j}} / \partial \mathrm{t}^{\mathrm{j}}\right)=\mathrm{H}_{0}^{\mathrm{j}} \mathrm{V}^{\mathrm{j}} \text {, }
$$

where $\mathrm{H}_{0}{ }^{\mathrm{j}}$ is the Hamiltonian operator in the $\mathrm{j}$-branch, and by summing on the branches:

$$
i \hbar \quad \Sigma_{j} a_{j}\left(\partial V^{j} / \partial t^{j}\right)=\Sigma_{j} a_{j} H_{0}^{j} V^{j} .
$$

One can recognize in the left side of this equation the action of the time-information derivative operator $\Delta_{\tau}$ on the vector $\mathrm{V}$ of $\mathrm{H}(\tau)$, multiplied by the constant $i \hbar \chi$, and in the right side the action of the extended Hamiltonian operator on this same vector. Thus, one obtains the following evolution law for a vector $V$ of the Hilbert space $H(\tau)$ :

$$
\text { i } \hbar \Delta_{\tau} \mathrm{V}=\mathrm{H} \mathrm{V}
$$

As in standard quantum mechanics, the Schrödinger-like evolution in $H(\tau)$ can be expressed more generally for mixtures, by using the definition of density operators and the algebra of operators in $H(\tau)$ (see section IV). The Liouville-von Neumann equation can thus be reformulated in $H(\tau)$ as:

$$
\text { i } \hbar \chi \Delta_{\mathrm{t}} \mathrm{D}=[\mathrm{H}, \mathrm{D}] \text {. }
$$


Moreover, the reformulation of the Schrödinger-like evolution in $\mathrm{H}(\tau)$ is of course possible for its relativistic generalizations. In this case, the Hilbert space $\mathcal{H}_{\mathrm{j}}$ associated with each possible history is a Fock space, that is, a sum of Hilbert spaces relative to each of the possible numbers of particles, while the extended Hamiltonian operator may contain extended creation and annihilation operators and relativistic terms.

\section{2. Age operator and information operator in IBU models.}

In order to deal with the dynamics of physical systems in IBU models, it is very convenient to specify, for a historical sate of $H(\tau)$, its possible (past) histories or "preparations", which can be regarded as computational paths in the universal branching structure, and, on the other hand, its information-structure content. This task can be realized by defining :

- an "age" operator acting on the historical state of any physical system (in the space $H(\tau)$ ) and whose action is to compute its informational "depth" which can be defined on the base of its preparation algorithmic complexity defined in section I,

- an information operator acting on the historical state of any physical system and whose action is to compute its encoding algorithmic complexity (also defined in section I) at $\tau$, via that of its "components" on each of the branches of its associated branching structure.

1) The action of the age operator $\Pi$ on a density operator $D=\Sigma_{j} \mu_{j} D^{j}$ of $H(\tau)$ is defined as follows:

$$
\Pi \mathrm{D}={ }_{\mathrm{df}} \quad \Sigma_{\mathrm{j}} \mu_{\mathrm{j}} \pi[\mathrm{j}] \mathrm{D}^{\mathrm{j}}
$$

where $\pi[j]$ is the informational depth of the computational path (or "preparation"), along the $\mathrm{j}$-branch, of the state $\mathrm{D}^{\mathrm{j}}$. The informational "depth" $\pi[\mathrm{j}]$ is defined according to Mora's and 
Briegel's notion of preparation algorithmic complexity of a quantum state: it is the minimal value, on all possible preparations $\mathrm{C}_{\varepsilon}^{\mathrm{B}}$ of $\mathrm{D}^{\mathrm{j}}$ built with gates of $\mathrm{B}$ that prepare $\mathrm{D}^{\mathrm{j}}$ with precision $\varepsilon$, of the classical algorithmic complexity of the series of symbols that encode the circuit $C^{B}{ }_{\varepsilon}$ according to a given code $\Omega^{7}$. In the above definition of $\Pi$, one has written $\pi[j]$ without specifying neither $\Omega$, B and $\varepsilon$ nor the specific state $\left(\mathrm{D}^{\mathrm{j}}\right.$ or $\mathrm{D}^{\mathrm{j}}$ or...) of the space $\mathcal{H}_{j}$ since 1) the choice of $\Omega$, B and $\varepsilon$ can be made once and for all and can then be omitted and 2) according to the construction of the universal branching structure (section II and III), the path $\mathrm{j}$ also prepares all other sub-states involved in the expression of the universal wave function at $\tau$-which are relative to other parts of the physical universe. Let us remind that, with the notations of section II, the wave function of the universe can be written as (focusing here on its “object"-part):

$$
\psi=\psi^{\mathrm{S} 1} \psi^{\mathrm{S} 2} \ldots \ldots \ldots \psi^{\mathrm{Sn}} \psi_{[\text {Imemory } ; \text { actions] }}^{\mathrm{IGUS}}
$$

where $\psi^{\mathrm{S} 1}, \psi^{\mathrm{S} 2} \ldots \ldots \psi^{\mathrm{Sn}}$ are the corresponding states of diverse parts of the physical universe for the same value $\tau$ of the time-information parameter.

$\Pi$ is hermitian since its eigenvalues are real numbers (indeed, they are integers):

$$
\Pi D^{j}=\pi[j] D^{j}
$$

and it can be easily verified that $\Pi$ is linear by using the fact that, as explained in the previous paragraph, the informational depth of all density operators of space $\mathcal{H}_{\mathrm{j}}$ (but not those of the different spaces $\mathcal{H}_{\mathrm{j}}$ ) has the same value $\pi[\mathrm{j}]$.

The average value of $\Pi$ in the state $D$ computes the average value of the informational depth of its possible preparations

7 The code $\Omega$ is, of course, supposed to be uniquely decidable, that is, the code-word of a path in the branching model is unique and can be read in an unambiguous manner. 


$$
<\Pi>_{\mathrm{D}}=\Sigma_{\mathrm{j}} \mu_{\mathrm{j}} \pi[\mathrm{j}],
$$

and gives thus an estimate, at time $\tau$, of the sate of development or of the "age" of the branching structure under consideration. Note that $\langle\Pi\rangle_{D}$ is a strictly positive quantity since $0 \leq \mu_{\mathrm{j}} \leq 1$ and $\pi[\mathrm{j}]>0$ for all $\mathrm{j}$. On the other hand, it can be written as:

$$
<\Pi>_{\mathrm{D}}=\operatorname{Tr} \Pi \mathrm{D} .
$$

2) The action of the information operator $K$ on a density operator $D=\Sigma_{j} \mu_{j} D^{j}$ of $H(\tau)$ is defined as follows :

$$
\mathrm{K} \mathrm{D}=\mathrm{df} \quad \Sigma_{\mathrm{j}} \mu_{\mathrm{j}} \mathrm{k}\left[\mathrm{D}^{\mathrm{j}}\right] \mathrm{D}^{\mathrm{j}}
$$

where $k\left[D^{j}\right]$ is the encoding algorithmic complexity of $D^{j}$.

Note that $\mathrm{K}$ is hermitian since its eigenvalues are real numbers:

$$
K D^{j}=k\left(D^{j}\right) D^{j}
$$

(but it can be easily verified that $\mathrm{K}$ is not linear).

The average value of $\mathrm{K}$ in the state $\mathrm{D}$ :

$$
<\mathrm{K}>_{\mathrm{D}}=\Sigma_{\mathrm{j}} \mu_{\mathrm{j}} \mathrm{k}\left[\mathrm{D}^{\mathrm{j}}\right]
$$

is also a strictly positive quantity which gives an estimate of the information encoded in the state D. It will be used in section V.4 for dealing with the thermodynamic asymmetry.

\section{3. A universal information-energy uncertainty relation.}

Focusing in this section exclusively on the Shrödinger-like evolution of physical systems, one can show an uncertainty relation between energy and "time"-information (featured by the total order T, defined in section III). This derivation is universal since it can apply to any physical system, disregarding the particular quantities that can be measured on it. This 
derivation follows the lines of the well known Mandelstam and Tamm's derivation (Mandelstam and Tamm 1945) .

By using the Schrödinger-like evolution law in $\mathrm{H}(\tau)$ shown in section V.1 and its conjugate, the fact that $\Pi$ and $\mathrm{H}$ are linear and that $\Pi$ does not depend explicitly on $\tau$, one can write the rate of change $\varpi_{\pi}$ of $<\Pi>_{\mathrm{D}}$ as :

$$
\left.\varpi_{\pi}=\Delta_{\tau}<\Pi>_{\mathrm{D}}=\operatorname{Tr} \Pi \Delta_{\tau} \mathrm{D}=(1 / \mathrm{i} \chi \hbar)<[\Pi, \mathrm{H}]\right\rangle_{\mathrm{D}} .
$$

On the other hand, using the facts that $\Pi$ and $H$ are hermitian and linear, one can show (according to a well known derivation) that the standard deviation $\Delta_{\mathrm{D}} \Pi$ of the age operator $\Pi$ in the state $D$, defined as usually (but in the space $H(\tau)$ ) by :

$$
\left(\Delta_{\mathrm{D}} \Pi\right)^{2}=_{\mathrm{df}}<\left(\Pi-<\Pi>_{\mathrm{D}}\right)^{2}>_{\mathrm{D}},
$$

and the standard deviation $\Delta_{\mathrm{D}} \mathrm{H}$ of the extended Hamiltonian operator $\mathrm{H}$ in state D satisfy the following uncertainty relation :

$$
\Delta_{\mathrm{D}} \Pi \Delta_{\mathrm{D}} \mathrm{H} \geq 1 / 2 \quad \mathrm{I}<[\Pi, \mathrm{H}]>_{\mathrm{D}} \mathrm{I} .
$$

Let us now define the change of the IGUS's memory size corresponding to a notable evolution of the system under consideration in state D:

$$
\delta_{\pi} \tau=\Delta_{\mathrm{D}} \Pi /\left|\varpi_{\pi}\right|
$$

This characteristic "duration"-information $\delta_{\pi} \tau$ is the change of the IGUS's memory size for which the average value of the age operator (that is, the "age" of the informational branching model) has changed by an amount equal to its standard deviation. It thus characterizes the growth of the branching model associated with the evolution of the system under consideration. Moreover, it does not rely on some particular "clock-observable" that could be defined on a particular system (Mandelstam and Tamm 1945) but its definition is 
universal: it can be used for evaluating the characteristic change of the IGUS's memory size corresponding to a notable evolution of any physical system.

Note that $\Delta_{\mathrm{D}} \Pi$ is a priori different from zero since the integers $\pi[j]$, which evaluate the computational depths of the preparations of the components $\mathrm{D}^{\mathrm{j}}$ of $\mathrm{D}$ according to different branches, are, a priori, mutually different. Moreover, one can see that, unless for the limiting case where $\mathrm{D}$ is a stationary state (in this case $\varpi \pi$ is equal to zero, and then $\delta_{\mathrm{K}} \tau$ is infinite but $\Delta_{\mathrm{D}} \mathrm{H}$ is equal to zero in conformity with the uncertainty relation (4) shown hereafter), $\delta_{\mathrm{K}} \tau$ is a finite quantity since it can be shown as follows that $[\Pi, \mathrm{H}]$ (and then $\left|\varpi_{\pi}\right|$ ) are different from 0 :

Suppose that $[\Pi, H]$ is equal to zero. Then, by the fact that the $D^{j}$ are eigenoperators of $\Pi$ with eigenvalues $\pi[j]$, the operators $\mathrm{H} \mathrm{D}^{\mathrm{j}}$ would be eigenoperators of $\Pi$ with the same eigenvalue $\pi[\mathrm{j}]$ :

$$
\Pi H D^{j}=\pi[j] H D^{j}, \text { for all } j,
$$

which can be written as :

$$
(\Pi-\pi[j] 1 H) H D^{j}=0, \text { for all } j,
$$

where $1 \mathrm{H}$ is the identity operator in the space $H(\tau)$. Consequently, by using the Liouville-von Neumann equation reformulated in $\mathrm{H}(\tau)$, one obtains that :

$$
\mathrm{i} \hbar(\Pi-\pi[\mathrm{j}] 1 \mathrm{H})\left[\mathrm{H}, \mathrm{D}^{\mathrm{j}}\right]=0 \text {, for all } \mathrm{j} \text {. }
$$

The operators $(\Pi-\pi[\mathrm{j}] 1 \mathrm{H})$ being different from the null-operator for all $\mathrm{j}$ (by the definition of $\Pi$ ), the previous equality is realized only if $\left[H, D^{j}\right]=0$ for all $j$, that is to say, if $[H, D]=0$, which expresses the fact that $\mathrm{D}$ is a stationary state - which is contrary to our hypothesis. 
Combining now the expressions (1) and (2) and the definition (3), one obtains a universal information/ energy uncertainty relation :

$$
\delta_{\pi} \tau \quad \Delta_{\mathrm{D}} \mathrm{H} \geq 1 / 2 \chi \hbar
$$

that is, a relation which is valid for any physical system (and, in the end, independent of the code $\Omega$, the gate basis B and the precision $\varepsilon$ that were used to evaluate the informational depth). This relation establishes, for any physical system, a link between its energy spread and the "growth" of its associated informational branching model, a growth which consists in the constant development of branches and the creation of new ones.

In the ideal case one can suppose the system really isolated, that is, if it is not submitted to measurements and its observables are not (or not any more) correlated with those of its environment, its associated IBU model is reduced to only one branch. In this case, $\Delta_{\mathrm{D}} \mathrm{H}$ reduces to $\Delta_{\rho} \mathrm{H}_{0}$, the standard deviation, in the non-branched state $\rho$, of the Hamiltonian operator of the Hilbert space $\mathrm{H}_{0}$ defined as usually for $\mathrm{S}$; on the other hand, the ratio $\left(\delta_{\pi} \tau / \chi\right)$ is a duration $\delta$ t of the linear time scale defined for this unique branch corresponding to a noticeable evolution (featured by the variation $\Delta_{\rho} \mathrm{H}_{0}$ ) of the system under consideration. Consequently, for this ideal case, the relation (4) reduces to Heisenberg's time/energy uncertainty relation:

$$
\delta \mathrm{t} \Delta \mathrm{H}_{0} \geq 1 / 2 \hbar
$$

One has thus provided a universal derivation of Heisenberg's time-energy uncertainty relation whereas, as is well known, no derivation of this relation in terms of standard deviations of Hamiltonian and time operators can be provided within the framework of standard quantum mechanics since no universal time operator can be defined (Pauli 1926). Indeed, if no external time operator can be defined, it remains possible, as shown by the present paper, to define an internal but nevertheless universal "age" operator whose standard 
deviation can be used to first derive a universal information/energy uncertainty relation and, as a special case, Heisenberg's time/energy uncertainty relation.

\section{4. The arrow of time in IBU models.}

Since Boltzmann's controversial attempt to explain the thermodynamic asymmetry from the time-symmetric, more "fundamental" laws of mechanics, the debate still remains open (Savitt 1995) (Price 1996). The numerous ideas that have been proposed to deal with the question of the thermodynamic "arrow of time" can be roughly classified in two categories. The first one is the statistical approach, initiated by Boltzman and Gibbs (Boltzmann 1910) (Gibbs 1902) and more recently developed by many authors, like Lebowitz (1999) or Balian (2005), according to which the thermodynamic asymmetry could be explained by the transition from the microscopic level of description of physical systems to their description in terms of the macroscopic variables that can be actually measured. The second one is the cosmological approach, initiated by Gold (1962) and Gal-Or (1972) and more recently developed by Penrose (1989), Hawking (1985), Zeh (1989) or Price (1996), according to which "the origin of irreversibility in all local processes as well as all time asymmetries observed in nature, can be traced back to the boundary conditions that gave rise to the expansion of the universe as a whole. The origin of irreversibility is thus not in the symmetrical laws of dynamics but in the boundary (or initial) conditions" (Gal Or 1972).

As emphasized by many authors since Boltzmann's proposal, the statistical approach suffers from the default of circularity which consists to implicitly assume the asymmetry we want to derive at the beginning of the derivation (Costa de Beauregard 1962) (Bitbol 1996, Appendix) (Price 1996, chap. 2). To be precise, the "right" direction of time according to which the second law of thermodynamics and any phenomenological law must be applied to 
agree with observation is not specified therein and cannot thus be defined from them. Consequently, a meta-theoretical principle must be set independently ("at the beginning of the derivation") to stipulate the direction of time (in fact, the usual "past-future" direction of life) for which these phenomenological laws must be applied in order to agree with what we observe.

Turning now to the cosmological approach, we are confronted with the two following questions:

(A) the question to explain why the universal "entropy is low near the Big Bang without thereby demonstrating that it must be low near a Big Crunch, in the event that the universe recollapses" (Price 1994 p.3). The question of the asymmetry of phenomena is thus replaced by that of the asymmetry between the "initial" and the "final" conditions for the whole universe.

Moreover, even if such an asymmetry can be established, the question of showing that these "initial" and "final" instants of cosmological time are, respectively, in our past and in our future is not resolved. In other words, the main difficulty of the statistical approach remains, that is to say:

(B) the question of the agreement between the past-future direction of life and the direction of time for which the entropy of an isolated system increases.

However, in IBU models an "anthropic"-like argument can be proposed to deal with this long standing debate: taking as primary the existence of IGUSes (endowed with an erasureresistant memory) that function asymmetrically, by acquiring (and not erasing) information on the world through measurements, questions (A) and (B) can find answers. For, in as much as in the evolving IBU models the splitting of histories is triggered by measurement operations, beside Shrödinger-like evolution any physical system is submitted to $a$ progressive loss of information-structure along its alternative histories whose number is 
constantly increasing. This loss of information-structure inherent to the branching dynamics can manifest in the two following cases:

- locally, if a measurement is performed on a physical system. In this case, the interference terms between the eigenvectors of the measured observable are rubbed out and, consequently, the state vector after the measurement is algorithmically more simple than the initial state vector (see section II and III).

- but also non locally, and this is the essential point, if measurements are performed on other systems whose observables have been correlated to its own during its preparation. Such quantum correlations "by the past" are a priori involved in the preparation of any physical system -and not only in the paradigmatic EPR experiment. For any physical system has been "prepared" in contact with the rest of the world and then some of its observables are necessarily correlated with those of its environment. As a consequence of the branching dynamics, which expresses the realization of measurements on the world -and, in particular, on the environment of this system-, the quantum correlations between this system and its environment are progressively destroyed. Consequently, the informational content of its state will decrease monotonically with $\tau$, the "time"-information parameter, until no more correlation exists with its environment -which thus defines an "equilibrium" state for this system. Thermodynamic irreversibility thus appears as a consequence of the branching process which takes place in the direction of increasing informational "time" and, consequently, in the direction of increasing time in any branch of its associated informational branching structure.

To clarify this essential point, let us consider an "isolated" system S whose state in US is $\mathrm{D}_{0}$ at time $\tau$. The corresponding memory state of the IGUS can be taken as the "root" of the branching structure $\mathbf{U}_{\mathbf{S}}$. That is to say, the representation space associated with $\mathbf{S}$ at $\tau$ is 
supposed to be the usual one-dimensional Hilbert space $\mathcal{H}$ and then the "initial" state $\mathrm{D}_{0}$ will not be decomposed in j-components.

In fact, as mentioned above, $\mathrm{S}$ results from a process of preparation that has begun before $\tau$. During this process of preparation some of its observables have been necessarily correlated to those of its environment $\mathrm{E}$ with which it has been in contact. Consequently, $\mathrm{S}$ must be considered as a part of the bigger system composed by $\mathrm{S}$ and its environment $\mathrm{E}$. Which means that, like in standard quantum mechanics, its state $\mathrm{D}_{0}$ can be regarded as the partial trace, on E, of the state $\mathrm{D}_{\mathrm{S}+\mathrm{E}}$ (defined in the appropriate extended Hilbert space) of the global system:

$$
\mathrm{D}_{0}=\operatorname{Tr}_{\mathrm{E}}\left(\mathrm{D}_{\mathrm{S}+\mathrm{E}}\right) .
$$

Now, by the very definition of IBU models, the "flow" of time-information between $\tau$ and $\tau^{\prime}>\tau$ results from the series of measurement operations that have been performed on physical systems and, in particular, on $E$ during the laps $\left(\tau^{\prime}-\tau\right)$. Assume that a measurement is performed on an observable A of E which is "initially"(that is, at $\tau$ ) strongly correlated with the observable B of $S$ with eigenvalues $b_{j}$, and that the effect of a measurement of $A$ on $E$ is to transform the "initial" state $\mathrm{D}_{0}$ into one of the states $\mathrm{D}^{\mathrm{j}}$, for some $\mathrm{j}$ :

$$
\mathrm{D} \rightarrow \mathrm{D}^{\mathrm{j}}=\mathrm{P}^{\mathrm{j}} \mathrm{D}_{0} \mathrm{P}^{\mathrm{j}} / \operatorname{Tr} \mathrm{D}_{0} \mathrm{P}^{\mathrm{j}}
$$

where $\mathrm{P}^{\mathrm{j}}$ is the projector of $\mathcal{H}_{\mathrm{j}}$ corresponding to the eigenvalue $\mathrm{b}_{\mathrm{j}}$ of $\mathrm{B}$.

In this $\mathrm{j}$-transformation, which is a reduction process, all the terms of $\mathrm{D}_{0}$ which involve the eigenvectors associated with $b_{j}$, for $j^{\prime} \neq j$, have been rubbed out. Consequently, disregarding the normalization term $\operatorname{TrDP}^{\mathrm{j}}$ which has no physical meaning, one can say that the encoding complexity of the microstates $D^{j}$ of $\mathcal{H}_{j}$ that result from the $j$-transformations is smaller than that of $\mathrm{D}_{0}$ (see section $\left.\mathrm{I}\right)$ :

$$
\mathrm{k}\left[\mathrm{D}^{\mathrm{j}}\right]<\mathrm{k}\left[\mathrm{D}_{0}\right] \text {, for all } \mathrm{j}
$$


This argument can of course be repeated for the series of transformations that constitute the $\mathrm{j}$-history of $\mathbf{U}_{\mathrm{S}}$, that is, according to the direction of increasing "time"-information:

$$
\mathrm{D}_{0} \rightarrow \mathrm{D}^{\mathrm{j}} \rightarrow \mathrm{D}^{\mathrm{j}} \longrightarrow \mathrm{D}^{\prime, j} \longrightarrow \ldots \ldots
$$

Along this sequence of $\mathrm{j}$-transformations, the average algorithmic information content of the j-“component" of the quantum state of S decreases monotonically with time :

$$
\mathrm{k}\left[\mathrm{D}_{0}\right]>\mathrm{k}\left[\mathrm{D}^{\mathrm{j}}\right]>\mathrm{k}\left[\mathrm{D}^{\mathrm{j} j}\right]>\mathrm{k}\left[\mathrm{D}^{, \mathrm{j}}\right] \quad>\ldots \ldots . .
$$

that can be written as :

$$
\boldsymbol{\Delta}_{\tau} \mathrm{k}\left[\mathrm{D}^{\mathrm{j}}\right] \leq 0, \text { along any } \mathrm{j} \text {-branch. }
$$

Using the definition of the average value of the information operator $\mathrm{K}$ :

$$
\langle\mathrm{K}\rangle_{\mathrm{D}}=\Sigma_{\mathrm{j}} \mu_{\mathrm{j}} \mathrm{k}\left[\mathrm{D}^{\mathrm{j}}\right] \text {, }
$$

it can be concluded that for any system $\mathrm{S}$, the average value of the information operator $\langle\mathrm{K}\rangle_{\mathrm{D}}$ (which is a positive quantity) can play the role of a thermodynamic potential for $S$, independently of the possible $\mathrm{j}$-branches involved in its evolution:

$$
<\mathrm{K}>_{\mathrm{D}}>0 \text { and } \quad \boldsymbol{\Delta}_{\tau}<\mathrm{K}>_{\mathrm{D}} \leq 0
$$

which expresses the fact that $\langle\mathrm{K}\rangle_{\mathrm{D}}$ decreases monotonically until no more correlation exist between $S$ and $E$-which thus defines the equilibrium state of $S$, corresponding to its genuine isolation.

A structural entropy can now be defined in order to characterize the progressive loss of information-structure of physical systems due to the branching dynamics:

$$
\sigma(\mathrm{D})=_{\mathrm{df}}\left\langle\mathrm{K}>_{\mathrm{Deq}_{\text {eq }}}-\delta<\mathrm{K}\right\rangle_{\mathrm{D}}
$$

where $\delta<\mathrm{K}\rangle_{\mathrm{D}}=\langle\mathrm{K}\rangle_{\mathrm{D}}-\langle\mathrm{K}\rangle_{\mathrm{D} \text { eq }}$ measures the difference between the average value of $K$ in the state $D$ and its average value in the equilibrium state $D_{e q}$.

From the definition (7) of the structural entropy $\sigma$ (D) and the property (6) one obtains that : 


$$
\sigma(\mathrm{D})>0 \quad \text { and } \quad \boldsymbol{\Delta}_{\tau} \sigma(\mathrm{D})=-\boldsymbol{\Delta}_{\tau}<\mathrm{K}>_{\mathrm{D}} \geq 0
$$

whereas the maximum value of the structural entropy for the equilibrium state is :

$$
\sigma_{\max }=\sigma\left(D_{\text {eq }}\right)=\langle K\rangle_{\text {D eq }} .
$$

Moreover, as can be shown from an important theorem of algorithmic information theory, on each branch $\mathrm{j}$ the equilibrium structural entropy $\sigma\left(\mathrm{D}_{\mathrm{eq}}^{\mathrm{j}}\right)$ can be identified to the equilibrium thermodynamic entropy of the system $S$ to within a (computer-dependant) constant:

$$
\sigma\left(D_{\text {eq }}^{j}\right)=\langle K\rangle_{D_{\text {eq }}^{j}}^{j_{1}} \cong S_{\text {thermo }}\left(D_{\text {eq }}^{j}\right)
$$

This identification comes from a fundamental double inequality according to which the ensemble average of the algorithmic information content of microstates of an ensemble $E$ of binary series $s_{\alpha}$ is bounded from below by the statistical entropy of that ensemble (expressed in "natural" units of information) and from above by the sum of the statistical entropy and the algorithmic information content of the whole ensemble ${ }^{8} E$ (Levin 1976) (Chaitin 1977) (Zurek 1989) (Caves 1990):

$$
\left.\mathrm{S}_{\text {stat }}(E) \leq<\mathrm{k}\left[\mathrm{s}_{\alpha}\right]\right\rangle_{\mathrm{E}} \leq \mathrm{S}_{\text {stat }}(\mathrm{E})+\mathrm{k}(\mathrm{E})+\mathrm{O}(1)
$$

where $\mathrm{O}(1)$ is a constant associated with the choice of the universal computer.

For thermodynamic ensembles on has $\mathrm{k}(\mathrm{E}) \ll \mathrm{S}_{\text {stat }}(\mathrm{E})$ since a few macroscopic parameters are necessary to compute the possible accessible states and their associated probabilities. Consequently, at the equilibrium state, this inequality reduces to an equivalence, always within a computer dependent constant, between the statistical entropy of the equilibrium state (and then of the equilibrium thermodynamic entropy) and the average value of the encoding

\footnotetext{
8 That is, the length of the shortest program that produces all the series $\mathrm{s}_{\mathrm{i}}$ and their associated probabilities.
} 
algorithmic complexity of its microstates (where the statistical ensemble is defined by the corresponding density operator $\rho$ whereas the series $\mathrm{s}_{\alpha}$ describe its eigenprojectors $\mathrm{P}_{\alpha}$ ):

$$
\mathrm{S}_{\text {thermo }}\left(\rho_{\text {eq }}\right)=S_{\text {stat }}\left(\rho_{\text {eq }}\right) \cong<\mathrm{k}\left[\mathrm{P}_{\alpha}\right]>\rho_{\text {eq }}
$$

This equivalence on which relies our proposal has been checked by Zurek in a concrete case (1989, Appendix A). This author has shown that the algorithmic information content of a typical microstate of a classical, ideal gas in thermodynamic equilibrium is equal to its thermodynamic entropy -which is given by the Sackur-Tetrode formula.

In IBU models, this equivalence can be applied for each of the branches to the corresponding quantities, by using the definition (7) of the structural entropy $\sigma$ and the definition of the average value of the information operator $\mathrm{K}$ :

$$
S_{\text {thermo }}\left(D_{\text {eq }}^{j}\right)=S_{\text {stat }}\left(D^{j}{ }_{\text {eq }}\right) \cong<K>_{D}^{j}{ }_{\text {eq }}=\sigma\left(D_{\text {eq }}^{j}\right) \text {. }
$$

Thus, it seems that the notion structural entropy defined by (7) can actually interpret that of thermodynamic entropy. According to this interpretation, the increase of thermodynamic entropy of a so-called "isolated" system is not a consequence of paradoxical "external perturbations" and it is not regarded as a progressive loss of (statistical) informationknowledge -which gives rise to a misleading, purely subjectivist interpretation of thermodynamic irreversibility (Szillard 1929) (Brillouin 1956) (Jaynes 1957) (Balian 1982).

Turning now to questions (A) and (B) mentioned at the beginning of this section, working in IBU models allows their following reformulation in terms of informational process :

- question (B), concerning the agreement between the "flow" of time, which brings us from our past to our future, and the direction of time for which the entropy of an isolated system increases. As shown above, in IBU models the monotonic increase of thermodynamic entropy for "isolated" systems relies on the spreading of information-structure along the branches of 
the evolving informational universe. Consequently, it takes place along the direction of increasing time-information parameter which defines the direction of the branching -and not the reverse. Now, if one can assume that the flow of informational "time" defined in this paper coincides with that of our familiar time of life, one can consider to have provided an answer to question (B). This coincidence between informational "time" and time of life can be justified by the fact that maintenance and development of life presuppose that, like for IGUSes, living beings be considered as "complex adaptive systems" (Gell-Man and Hartle 1989) that function by optimally gathering and utilizing information about the physical world. Moreover, at a higher level of development, the construction of the human symbolic system ${ }^{9}$ requires an incessant process of information exchange between the subject of knowledge and action (the human community) and its environment. Living beings taking part to such an information process are modelled in this paper, in a rudimentary way, by IGUSes that function by performing measurements on the world, recording their outcomes and planning their subsequent actions (measurements).

- question (A), concerning the asymmetry between "initial" and "final" conditions for the universe. In IBU models, the notions of initial and final conditions (or that of boundary conditions) for a strongly "objective" universe whose existence is independent of the presence of observers has no place. For, the states of the informational universe associated with a physical system are defined as states of knowledge which are fundamentally observerdependant. However, IBU models are asymmetric and this asymmetry, which does not rely on some speculative, ad-hoc extra-ingredient regarding the independent reality, finds its origin in the only informational process which involves the existence of IGUSes that function as described in this paper. Consequently, no initial (or final) conditions for the universe must be

9 The human symbolic system can be roughly defined as the entanglement of all cultural forms (language, myth, art, religion, science) in which and by which any experience appears to be significant for him (Cassirer 1972). 
set from an external, strongly objective point of view (as, for example, the very low "initial" value of the Weyl curvature which characterizes the properties of distortion of space-time due to gravity (Penrose 1989, chap. 7)) but the condition of low entropy of the remote "past" of the universe and that of the higher entropy of its "future" appear as mere consequences of the informational branching dynamics : This informational process takes place according to the increasing direction of time (in all branches) and, as shown above, it is responsible of a progressive loss of information-structure (and thus a monotonic increase of thermodynamic entropy) of any isolated system until its equilibrium state for which no more correlation exist with the rest of the universe. Moreover, if we admit that the whole universe can be analyzed as the reunion of parts that have been correlated because of their interaction during their preparation and that weakly interact (or do not interact any more) now, one can infer that the whole universe, like any of its parts, would have been submitted to a monotonic loss of information-structure with increasing time.

Thus, if one takes as primary, like in IBU models, the existence of IGUSes and the universal informational process they participate in, one can explain that the universe comes out from states of lower and lower structural entropy and evolves toward states of higher and higher structural entropy ${ }^{10}$-which constitutes, of course, a weaker statement of the required cosmological asymmetry expressed in question (A).

Generally speaking, the previous explanation of the thermodynamic asymmetry leads to the idea that the privileged directionality of all phenomena we observe would be a consequence of the branching dynamics of the evolving informational branching universe. For, as shown by many authors (Zeh 1989) (Gal-Or 1983), all time asymmetries observed in

10 The lower entropy of the past and the higher entropy of the future thus appears to be a necessary condition for the existence of IGUSes and their knowledge activity. This can be considered as an anthropic argument. 
nature -like the cosmological asymmetry defined by the expansion of the universe or the radiative asymmetry according to which only retarded waves can be observed- are strongly connected with the thermodynamic asymmetry. In other words, according to our proposal, the so-called "arrow of time" would be the necessary counterpart of the universal information process underlying the subject's activity of measuring - a process which defines the "flow" of time-information.

\section{Acknowledgements}

I am grateful to the organizers of the Kraków workshop on Branching Space-Times (October 2005) for the opportunity to present this work and to all participants of this workshop for interesting discussions. 


\section{Appendix}

Let $\mathrm{L}$ a first-order language that contains a symbol of binary relation, noted as $\mathbf{R}$.

IBU models are tree-like structures indexed by the values $\tau$ of the "time"-information linear scale defined in section III :

$$
\mathbf{U}(\tau)=\langle E, \mathbf{R}>,
$$

where $E$ is a discrete, non empty set whose elements are intuitively interpreted by the IGUS's memory states. For convenience, its elements will be noted below as x, y, z...

$\mathbf{R}$ is a strict, partial ordering relation that satisfies the following axioms :

Irreflexivity: $\forall \mathrm{x}\rceil(\mathrm{x} \mathbf{R} \mathrm{x})$

Transitivity: $\forall(\mathrm{x}, \mathrm{y}, \mathrm{z}) \in \mathrm{E}^{3}(\mathrm{x} \mathbf{R} \mathrm{y}) \wedge(\mathrm{y} \mathbf{R} \mathrm{z}) \Rightarrow(\mathrm{x} \mathbf{R} \mathrm{z})$

Partial ordering : $\left.\left.\exists(\mathrm{x}, \mathrm{y}) \in \mathrm{E}^{2}\right\rceil(\mathrm{x} \mathbf{R} \mathrm{y}) \wedge\right\rceil(\mathrm{y} \mathbf{R} \mathrm{x})$

Discretness : $\forall \mathrm{x}(\exists \mathrm{y}(\mathrm{y} \mathbf{R} \mathrm{x}) \Rightarrow \exists \mathrm{y}(\mathrm{y} \mathbf{R} \mathrm{x} \wedge\rceil \exists \mathrm{z}(\mathrm{y} \mathbf{R} \mathrm{z} \wedge \mathrm{z} \mathbf{R} \mathrm{x})))$

$$
\wedge \forall \mathrm{x}(\exists \mathrm{y}(\mathrm{x} \mathbf{R} \mathrm{y}) \Rightarrow \exists \mathrm{y}(\mathrm{x} \mathbf{R} \mathrm{y} \wedge\rceil \exists \mathrm{z}(\mathrm{x} \mathbf{R} \mathrm{z} \wedge \mathrm{z} \mathbf{R} \mathrm{y}))
$$

Moreover, $\mathbf{R}$ satisfies the specific tree properties:

Linearity of the "past": $\forall(\mathrm{x}, \mathrm{y}, \mathrm{z}) \in \mathrm{E}^{3}[(\mathrm{y} \mathbf{R} \mathrm{x}) \wedge(\mathrm{z} \mathbf{R} \mathrm{x})] \Rightarrow[(\mathrm{y} \mathbf{R} \mathrm{z}) \vee(\mathrm{z} \mathbf{R} \mathrm{y}) \vee(\mathrm{y}=\mathrm{z})]$ Connectivity: $\forall \mathrm{x} \forall \mathrm{y} \exists \mathrm{z} \quad(\mathrm{z} R \mathrm{x}) \wedge(\mathrm{zR} \mathrm{y})$.

A branch of the IBU model $U(\tau)$ is a subset of $E$ totally ordered by $R$ and maximal. The algorithmic informational content I(x) of the IGUS's memory states of a branch $b_{j}$ of $U(\tau)$ is smaller than $\tau: \forall \mathrm{x} \in \mathrm{b}_{\mathrm{j}} \quad \mathrm{I}(\mathrm{x}) \leq \tau$. 


\section{References}

Balian, R. (1982) Du Microscopique au Macroscopique. Cours de Physique Statistique de l'Ecole Polytechnique. Tome 1. Ellipses.

Balian R. (2005) "Information in statistical physics". Studies in Histories and Philosophy of Modern Physics 36.

Belnap, N. (2002) EPR-like "funny business" in the theory of branching space-times. http:// philsci-archive.pitt.edu

Belnap, N. (2003) Branching Space-Time. Post-print of Belnap 1992. http:// philsci-archive.pitt.edu

Belnap, N. and Szabó L. E. (1996) Branching Space-time analysis of the GHZ theorem. Foundations of Physics, arXiv:quant-ph/95 10002 v3 4 Jun 1996.

Bitbol, M. (1988) The concept of measurement and time symmetry in quantum mechanics. Philosophy of Science 55, p. 349-375.

Bitbol, M. (1996) Mécanique Quantique. Une introduction philosophique. Flammarion.

Boltzmann, L. (1910) Vorlesungen über Gastheorie. Barth, Leipzig.

Bohm, D. and Hiley, B. J. (1993). The Undivided Universe. Routledge.

Burgess, J. P. (1978) The unreal future. Theoria $n^{\circ} 4$, p. 157-179.

Brillouin, L. (1956) : Science and Information Theory. Academic Press. New York. 
Carnap, R. (1966) Philosophical Foundations of Physics. Chap. 24, ed. Martin Gardner, French translation Armand Colin 1973, Paris.

Caves, C.M. (1990) Entropy and Information : How much information is needed to assign a probability? In Complexity, Entropy and the Physics of Information, Zurek. W.H. (ed.), Addisson Wesley Publishing Company.

Chaitin, G.J. (1977) Algorithmic information theory. IBM J. Res. Develop. $\mathrm{n}^{\circ} 21$.

Clifton, R. , Bub, J. ,and Halvorson, H. (2003) Characterizing quantum theory in terms of information theoretic constraints. Foundations of Physics 33 (11), p. 1561.

Costa de Beauregard, O. (1963) Le Second Principe de la Science du Temps. Edition Seuil.

Destouche-Février, P. (1945) Sur l'impossibilité d'un retour au déterminisme en microphysique. Compte rendu de l'Académie des Sciences. T. 220, p. 587.

De Witt B. (1970) Quantum mechanics and reality. Physics Today, Vol. 23, n 9.

Deutsch, D. (1999) Quantum Theory of Probability and Decisions. Proceedings of the Royal Society of London A455, 3129-3137. http://www.arXiv.org/abs/quant-ph/9906015.

Espagnat, B. (d’) (1994) Le Réel Voilé. Analyse des concepts quantiques. Edition Fayard.

Espagnat, B. (d') (1979) The Quantum Theory and Reality. Scientific American 241, 5, Nov. 1979, p. 128-140.

Everett, H. (1957) The 'relative state" formulation of quantum mechanics. Review of Modern Physics 29, n 3, p. 454-462.

Gács P. (2001) Quantum Algorithmic Entropy. arXiv : quant-ph / 0011046 v2 .

Gal-Or B. 1983 : Cosmology, Physics and Philosophy. Springer-Verlag 1983. Part II. Lecture IV: "The Arrow of Time". 
Gell-Mann, M. and Hartle, J. B. (1990) Quantum Mechanics in the light of Quantum Cosmology. In Complexity, Entropy and the Physics of Information, Zurek. W.H. (ed.), Addisson Wesley Publishing Company.

Gibbs J.W. (1902) : Elementary Principles in Statistical Mechanics. Yale University Press.

Girhardi G. C., Rimini, A., Weber, T. (1986) Unified dynamics for microscopic and macroscopic systems. Physical Review C, 34, $n^{\circ} 2, p .470-491$.

Griffits, R. J. Consistent Histories and the Interpretation of Quantum Mechanics. Journal of Statistical Physics 36, p. 219.

Grinbaum, A. (2004) The Significance of Information in Quantum Theory. Thèse de Doctorat de l'Ecole Polytechnique, Paris.

Hawking, S. (1985) Arrow of time in cosmology. Physical Review D, Vol. 32, $n^{\circ}$ 10, p. 2489.

Jaynes; E. T. (1957) Information Theory and Statistical Mechanics. Phys. Rev. $n^{\circ} 2$ p. 171 and $n^{\circ} 4$ p. 620.

Levin, L. A. (1976) Various Measures of Complexity for Finite Objects Axiomatic Description. Soviet Math. Dokl. Vol 17, p. 522. (English translation)

Mandelstam, L.I. and Tamm, I. (1945) The uncertainty relation between energy and time in non-relativistic quantum mechanics. J. Phys. (USSR) $\mathrm{n}^{\circ} 9$.

Mc Call, S. (1998) "The combining of quantum mechanics and relativity theory." http://scistud.umkc.edu/psa98/papers/mccall.pdf

Mora C., Briegel H.J. (2004) "Algorithmic complexity of quantum states.“ ArXiv:quant-ph/0412172 v1 22 Dec 2004

Müller, T. (2001) Branching Space-Time, Modal Logic and the Counterfactual Conditional. http:// philsci-archive.pitt.edu 
Müller, T. (2005) Probability Theory and Causation: A Branching Space-Times Analysis. British Journal for the Philosophy of Science 56, p. 487-520.

Nielsen, M. A. (1998) Quantum Information Theory. Ph D Dissertation, University of New Mexico, Albuquerque, New Mexico, USA.

Omnès R. (2001) Probabilities, Decohering Histories, and the Interpretation of Quantum Mechanics, Lecture Notes in Physics, ed. Bricmont et al., vol. 574, p. 149.

Pauli, W. (1926) Handbuch der Physik. Vol 24/1, p. 143.

Penrose R. (1989) The Emperor's New Mind. Oxford University Press.

Placek, T. (2003) On objective transition probabilities. http://www.uni-konstanz.de/ppm/gap5workshop/Placek.pdf

Price, H. (1996) Time's Arrow and Archimede's Point. Oxford University Press.

Prior, A.N. (1967) Past, Present and Future. Clarendon Press.

Saunders, S. (1998) Time, quantum mechanics and probability. Synthese 114, p. 373-404.

Savitt, S. F. (1995) Time's Arrow Today. Cambridge University Press.

Schumacher, B. (1990) Information from Quantum Measurements. In Complexity, Entropy and the Physics of Information, Zurek. W.H. (ed.), Addisson Wesley Publishing Company.

Shannon C.E. and Weaver W. (1949) The Mathematical Theory of Communication. Ed. Urbana. University of Illinois Press.

Sharlow, M. F. (2004) What Branching Spacetime Might Do for Physics . http://www.eskimo.com/ msharlow 
Szillard, L. (1929) On the Decrease of Entropy in a Thermodynamic System by the Intervention of Intelligent Beings. Zeit. Phys. Vol. 53, p. 840. English translation in Quantum Theory and Measurement. Ed. Wheeler J. A. and Zurek W. H. Princeton University Press 1983.

Vaidman, L. (1998) On Schizophrenic Experiences of the Neutron or Why We should Believe in the Many-Worlds Interpretation of Quantum Theory. International Studies in the Philosophy of Science 12, 245-261.

Vitányi P. (2001) Quantum Kolmogorov Complexity Based on Classical Descriptions. IEEE Transactions on Information Theory, Vol. 47, No. 6, p. 2464-2479

Wallace, D. (2003) Everettian Rationality: defending Deusch's approach to probability in the Everett interpretation. http://users.ox.ac.uk/ mert0130/papers/decshort.pdf.

Wheeler, J. A; (1990) Information, Physics, Quantum : The Search for Links. In Complexity, Entropy and the Physics of Information, Zurek. W.H. (ed.), Addisson Wesley Publishing Company.

Wheeler, J. A and Zurek, W.H. (1983) Quantum Theory and Measurement. Princeton University Press.

Zeh, H. D. (1989) The Physical Basis of the Direction of Time. Springer Verlag.

Zurek, W. H. (1989) Algorithmic randomness and physical entropy. Phys. Rev. 40, n 40, p. 4731-4751.

Zurek, W. H. (2003) Decoherence, Einselection and the Quantum Origins of the Classical. arXiv quant-ph / 0105127v3. 